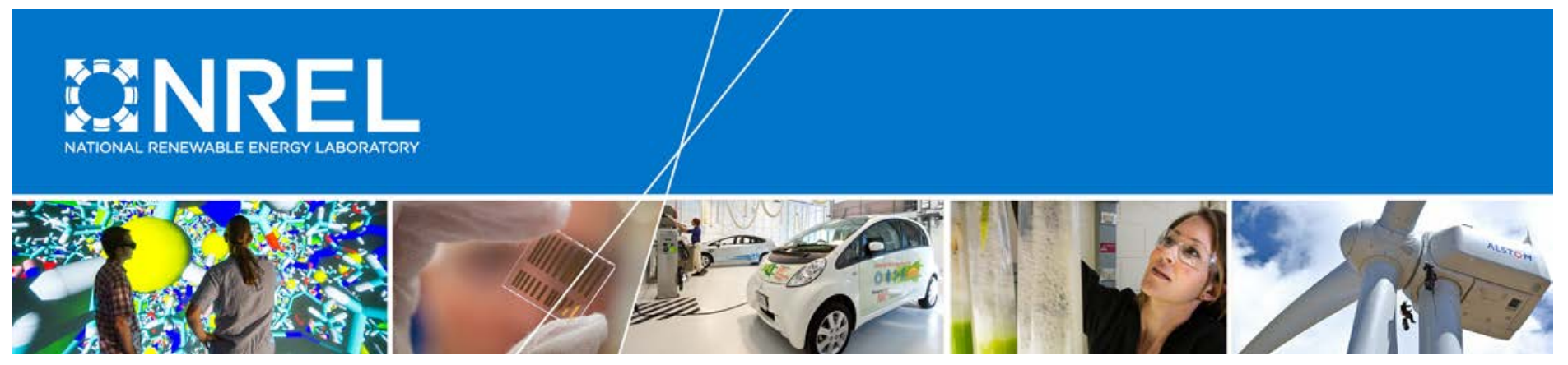

\title{
SubDyn User's Guide and Theory Manual
}

Rick Damiani, Jason Jonkman, and Greg Hayman National Renewable Energy Laboratory

NREL is a national laboratory of the U.S. Department of Energy Office of Energy Efficiency \& Renewable Energy Operated by the Alliance for Sustainable Energy, LLC

This report is available at no cost from the National Renewable Energy Laboratory (NREL) at www.nrel.gov/publications.

\section{Technical Report}

NREL/TP-5000-63062

September 2015 


\title{
SubDyn User's Guide and Theory Manual
}

\author{
Rick Damiani, Jason Jonkman, \\ and Greg Hayman \\ National Renewable Energy Laboratory \\ Prepared under Task No. WE14.5C01
}

NREL is a national laboratory of the U.S. Department of Energy Office of Energy Efficiency \& Renewable Energy Operated by the Alliance for Sustainable Energy, LLC

This report is available at no cost from the National Renewable Energy Laboratory (NREL) at www.nrel.gov/publications.

National Renewable Energy Laboratory 15013 Denver West Parkway Golden, CO 80401

303-275-3000 • www.nrel.gov

\section{Technical Report}

NREL/TP-5000-63062

September 2015

Contract No. DE-AC36-08GO28308 


\title{
NOTICE
}

This report was prepared as an account of work sponsored by an agency of the United States government. Neither the United States government nor any agency thereof, nor any of their employees, makes any warranty, express or implied, or assumes any legal liability or responsibility for the accuracy, completeness, or usefulness of any information, apparatus, product, or process disclosed, or represents that its use would not infringe privately owned rights. Reference herein to any specific commercial product, process, or service by trade name, trademark, manufacturer, or otherwise does not necessarily constitute or imply its endorsement, recommendation, or favoring by the United States government or any agency thereof. The views and opinions of authors expressed herein do not necessarily state or reflect those of the United States government or any agency thereof.

This report is available at no cost from the National Renewable Energy Laboratory (NREL) at www.nrel.gov/publications.

Available electronically at SciTech Connect http:/www.osti.gov/scitech

Available for a processing fee to U.S. Department of Energy and its contractors, in paper, from:

\author{
U.S. Department of Energy \\ Office of Scientific and Technical Information \\ P.O. Box 62 \\ Oak Ridge, TN 37831-0062 \\ OSTI http://www.osti.gov \\ Phone: 865.576.8401 \\ Fax: 865.576.5728 \\ Email: reports@osti.gov
}

Available for sale to the public, in paper, from:

\author{
U.S. Department of Commerce \\ National Technical Information Service \\ 5301 Shawnee Road \\ Alexandria, VA 22312 \\ NTIS http://www.ntis.gov \\ Phone: 800.553 .6847 or 703.605 .6000 \\ Fax: 703.605.6900 \\ Email: orders@ntis.gov
}




\section{Preface}

This manual offers both a quick reference guide and a more in-depth theory guide for the SubDyn software program. It is intended to be used by the general user in combination with the FAST and HydroDyn manuals. The manual will be updated as new releases are issued and as needed to provide further information on advancements or modifications to the software. 


\section{Acknowledgments}

The authors would like to acknowledge Huimin Song, a post-doctoral researcher at the National Renewable Energy Laboratory, for her contribution to the development of SubDyn. We also are grateful to the U.S. Department of Energy Wind and Water Power Program for supporting the development of this software. 


\section{Nomenclature}

$\Omega_{m}$

v

A

$A_{s x}$

$A_{s y}$

$A_{z}$

C-B

C

$d t \quad$ Time step in the integration algorithm

$d t_{\max } \quad$ Maximum time-step recommended

$D_{i}$

$D_{o}$

$D_{1}$

$D_{2}$

DOF

Eigenfrequency $(\mathrm{rad} / \mathrm{s})$

Material density $\left[\mathrm{kg} / \mathrm{m}^{3}\right]$ motion of the boundary nodes eigenmodes

Poisson's ratio

Member shear area along local $x$-axis

Member shear area along local $y$-axis

Craig Bampton

System damping matrix

Member inner diameter

Member outer diameter

Degree of Freedom
Damping ratio (percent of critical) or diagonal damping matrix

$L \times L$ matrix representing the $\mathrm{C}-\mathrm{B}$ eigenmodes

$L \times m$ matrix representing the retained $\mathrm{C}-\mathrm{B}$ eigenmodes

$L \times R$ matrix representing the interior nodal displacements for a static, rigid-body

Matrix representing the interior nodal displacements for a static, rigid-body motion of the boundary nodes, after the restrained DOFs are removed $L \times L$ diagonal matrix representing the eigenfrequencies of all C-B eigenmodes $L \times m$ diagonal matrix representing the eigenfrequencies of the retained C-B

State matrix in state-space state equation

Member cross-sectional area, normal to local $z$-axis

Input matrix in state-space state equation

State matrix in state-space output equation for ElastoDyn

State matrix in state-space output equation for HydroDyn

Damping matrix partition referred to the $L$ interior DOFs $(L \times L)$

Damping matrix partition referred to the $L$ and $R$ DOFs $(L \times R)$

Damping matrix partition referred to the $R$ and $L$ boundary DOFs $(R \times L)$

Damping matrix partition referred to the $R$ boundary DOFs $(R \times R)$

HydroDyn's linear damping matrix heave element

Input transmission matrix in state-space output equation for ElastoDyn

Input transmission matrix in state-space output equation for HydroDyn 


\begin{tabular}{|c|c|}
\hline$\left[\boldsymbol{D}_{C}\right]$ & $\begin{array}{l}\text { Member direction cosine } 3 \times 3 \text { matrix, transforming local coordinates to global } \\
\text { coordinates }\end{array}$ \\
\hline E & Young's modulus \\
\hline$f$ & Frequency \\
\hline$f_{\max }$ & Maximum frequency \\
\hline$F$ & Vector of external forces and moments \\
\hline$F_{G}$ & Array of self-weight forces and moments \\
\hline$F_{L}$ & Array of external (hydrodynamic) forces and moments at the interior nodes \\
\hline$\tilde{F}_{L}$ & $\begin{array}{l}\text { Interior forces and moments }(L \times 1) \text { array accounting for all interior modes }= \\
\Phi_{L}^{T}\left(F_{L}+F_{L g}\right)\end{array}$ \\
\hline$F_{L g}$ & Array of self-weight gravity forces and moments at the interior nodes \\
\hline$\tilde{F}_{m}$ & $\begin{array}{l}\text { Interior forces and moments }(L \times 1) \text { array accounting for only the retained modes }= \\
\Phi_{m}^{T}\left(F_{L}+F_{L g}\right)\end{array}$ \\
\hline$F_{R}$ & Array of external (hydrodynamic) forces and moments at the boundary nodes \\
\hline $\bar{F}_{R}$ & Array of external (hydrodynamic) forces and moments at the interface nodes \\
\hline$F_{\text {React }}$ & $\begin{array}{l}\text { Substructure base reaction forces and moments, applied to the substructure by the } \\
\text { restraint }\end{array}$ \\
\hline$F_{R g}$ & Array of self-weight forces and moments at the boundary nodes \\
\hline $\bar{F}_{R g}$ & Array of self-weight forces and moments at the interface nodes \\
\hline$F_{T P}$ & $\begin{array}{l}\text { TP reaction forces and moments, applied to the substructure by the remainder of } \\
\text { the turbine system }\end{array}$ \\
\hline$\tilde{F}_{T P}$ & TP forces and moments after C-B reduction, $=F_{T P}+T_{I}^{T} \bar{F}_{R g}+T_{I}^{T} \bar{\Phi}_{R}^{T}\left(F_{L}+F_{L g}\right)$ \\
\hline$F_{X}$ & $\begin{array}{l}\text { Substructure base reaction force along global } X \text {-axis; or } \\
\text { Forcing vector in state-space state equation }\end{array}$ \\
\hline$F_{Y}$ & Substructure base reaction force along global $Y$-axis \\
\hline$F_{Y 1}$ & Forcing vector in state-space output equation for ElastoDyn \\
\hline$F_{Y 2}$ & Forcing vector in state-space output equation for HydroDyn \\
\hline$F_{Z}$ & Substructure base reaction force along global $Z$-axis \\
\hline$F_{I}^{e}$ & Element inertia forces \\
\hline$F_{S}^{e}$ & Element static forces \\
\hline FEA & Finite-element analysis \\
\hline FEM & Finite-element method \\
\hline$g$ & Gravity, unsigned magnitude \\
\hline G & Shear modulus \\
\hline$i$ & Member or element index \\
\hline$\hat{i}_{e}$ & Unit vector along the element local $x$-axis \\
\hline
\end{tabular}




\begin{tabular}{|c|c|}
\hline$I$ & Identity matrix \\
\hline$\hat{I}$ & Unit vector along the global $X$-axis \\
\hline$\hat{j}_{e}$ & Unit vector along the element local $y$-axis \\
\hline$J$ & Generic second area moment of inertia \\
\hline$\hat{J}$ & Unit vector along the global $Y$-axis \\
\hline$J_{x}$ & Second area moment of inertia about the local, principal $x$-axis \\
\hline$J_{x x}$ & Second area moment of inertia about the local $x$-axis \\
\hline$k$ & Element stiffness matrix $(12 \times 12)$ in global coordinate system \\
\hline$k_{e}$ & Element stiffness matrix $(12 \times 12)$ \\
\hline$\hat{k}_{e}$ & Unit vector along the element local $z$-axis \\
\hline$k_{a x}$ & Shear area factor along local $x$-axis \\
\hline$k_{a y}$ & Shear area factor along local $y$-axis \\
\hline K & System stiffness matrix \\
\hline$\hat{K}$ & Unit vector along the global $Z$-axis \\
\hline$K_{B B}$ & Matrix partition after C-B system reduction $=K_{R R}+K_{R L} \Phi_{R}$ \\
\hline $\bar{K}_{B B}$ & $K_{B B}$ after removal of constrained DOF rows and columns \\
\hline$\tilde{K}_{B B}$ & $\begin{array}{l}\text { Substructure equivalent stiffness matrix referred to the TP reference point, = } \\
T_{I}^{T} \bar{K}_{B B} T_{I}\end{array}$ \\
\hline$K_{L L}$ & Stiffness matrix partition referred to the $L$ interior DOFs $(L \times L)$ \\
\hline$K_{L R}$ & Stiffness matrix partition referred to the $L$ and $R$ DOFs $(L \times R)$ \\
\hline$K_{R L}$ & Stiffness matrix partition referred to the $R$ and $L$ boundary DOFs $(R \times L)$ \\
\hline$K_{R R}$ & Stiffness matrix partition referred to the $\mathrm{R}$ boundary DOFs $(R \times R)$ \\
\hline$K_{s x}$ & Shear correction factor along local $x$-axis \\
\hline$K_{s y}$ & Shear correction factor along local $y$-axis \\
\hline$K_{33}^{(S D)}$ & Substructure equivalent heave stiffness \\
\hline$L$ & Interior nodes' DOFs \\
\hline LFEB & Linear frame finite-element beam model \\
\hline$L_{e}$ & Length of element \\
\hline$L_{e x y}$ & Length of element projection in the global $X Y$ plane \\
\hline$m_{e}$ & Element stiffness matrix $(12 \times 12)$ \\
\hline$m$ & $\begin{array}{l}\text { Element stiffness matrix ( } 12 \times 12) \text { in global coordinate system; or } \\
\text { number of retained C-B modes }\end{array}$ \\
\hline$M$ & System mass matrix \\
\hline MSL & Mean sea level \\
\hline$M_{B B}$ & Matrix partition after C-B system reduction $={ }_{M_{R R}}+M_{R L} \Phi_{R}+\Phi_{R}^{T} M_{L R}+\Phi_{R}^{T} M_{L L} \Phi_{R}$ \\
\hline $\bar{M}_{B B}$ & $M_{B B}$ after removal of constrained DOF rows and columns \\
\hline
\end{tabular}




\begin{tabular}{|c|c|}
\hline$\tilde{M}_{B B}$ & Substructure equivalent mass matrix referred to the TP reference point $=T_{I}^{T} \bar{M}_{B B} T_{I}$ \\
\hline$M_{B m}$ & Matrix partition after C-B system reduction $=M_{m B}^{T}$ \\
\hline $\bar{M}_{B m}$ & $M_{B m}$ after removal of constrained DOF rows and columns \\
\hline$\tilde{M}_{B m}$ & Matrix partition $=T_{I}^{T} \bar{M}_{B m}$ \\
\hline$M_{L L}$ & Mass matrix partition referred to the $L$ interior DOFs $(L \times L)$ \\
\hline$M_{L R}$ & Mass matrix partition referred to the $L$ and $R$ DOFs $(L \times R)$ \\
\hline$M_{m B}$ & Matrix partition after C-B system reduction $=\Phi_{m}^{T} M_{L R}+\Phi_{m}^{T} M_{L L} \Phi_{R}$ \\
\hline$\tilde{M}_{m B}$ & Matrix partition $=\tilde{M}_{B m}^{T}$ \\
\hline$M_{R L}$ & Mass matrix partition referred to the $R$ and $L$ boundary DOFs $(R \times L)$ \\
\hline$M_{R R}$ & Mass matrix partition referred to the $R$ boundary DOFs $(R \times R)$ \\
\hline$M_{X}$ & Substructure base reaction moment along global $X$-axis \\
\hline$M_{Y}$ & Substructure base reaction moment along global $Y$-axis \\
\hline$M_{Z}$ & Substructure base reaction moment along global $Z$-axis \\
\hline$M^{(E D)}$ & $6 \times 6$ mass matrix from ElastoDyn \\
\hline$M_{33}^{(S D)}$ & Substructure equivalent heave mass \\
\hline$n$ & The $\mathrm{n}^{\text {th }}$ time step \\
\hline NIN & Number of interface nodes \\
\hline$N_{\text {react }}$ & Number of restrained nodes \\
\hline$p$ & State-space parameters \\
\hline$q_{L}$ & Modal coefficients for all interior nodes' DOF modes \\
\hline$q_{L 0}$ & $\begin{array}{l}\text { Modal coefficients for all interior nodes' DOF modes assumed operating in static } \\
\text { fashion }\end{array}$ \\
\hline$q_{m}$ & Modal coefficients for the retained modes \\
\hline$q_{m 0}$ & Modal coefficients for the retained modes assumed operating in static fashion \\
\hline$R$ & Boundary nodes' DOFs \\
\hline$\vec{R}$ & Reaction forces at the base of the substructure \\
\hline$t$ & $\begin{array}{l}\text { time; or } \\
\text { thickness }\end{array}$ \\
\hline ТP & Transition piece \\
\hline$T_{I}$ & Matrix to transform interface nodes' DOFs to TP DOFs; $(6 \cdot N I N) \times 6$ matrix \\
\hline$T_{\text {react }}$ & $\begin{array}{l}\text { Auxiliary matrix }\left(6 \times\left(6 \cdot N_{\text {react }}\right)\right) \text { to link restrained nodes' forces and moments to the } \\
\text { substructure base reaction vector }\end{array}$ \\
\hline$u, u_{i}$ & State-space formulation inputs, generic i-th input \\
\hline$U$ & Vector of nodal displacements \\
\hline$U_{e}$ & Vector of element nodes' displacements (DOFs) \\
\hline
\end{tabular}




\begin{tabular}{|c|c|}
\hline$U_{L}$ & Vector of interior nodes' displacements (DOFs) \\
\hline$\hat{U}_{L}$ & Time-varying components of the interior nodes' displacements (DOFs) \\
\hline$U_{L 0}$ & Static components of the interior nodes' displacements (DOFs) $(L \times 1)$ \\
\hline$U_{L 0 m}$ & Static components of the interior nodes' displacements (DOFs) $(L \times 1)$, but \\
\hline$U_{R}$ & $\begin{array}{l}\text { obtained considering the first } m \text { C-B eigenmodes only } \\
\text { Vector of boundary nodes' displacements (DOFs) }\end{array}$ \\
\hline $\bar{U}_{R}$ & Vector of interface nodes' displacements (DOFs) \\
\hline$U^{e}$ & Element nodes' displacements (DOFs) \\
\hline$\hat{U}^{e}$ & Time-varying components of the element nodes' displacements (DOFs) \\
\hline$U_{L 0}^{e}$ & Static components of the element nodes' displacements (DOFs) \\
\hline$U_{L 0 m}^{e}$ & Static components of the element nodes' displacements (DOFs), but obtained \\
\hline$U_{T P}$ & $\begin{array}{l}\text { considering the first } m \text { C-B eigenmodes only } \\
\text { TP reference point displacements (DOFs) }\end{array}$ \\
\hline$\hat{U}_{T P}$ & Time-varying components of the TP reference point displacements (DOFs) \\
\hline$U_{T P 0}$ & Static components of the TP reference point displacements (DOFs) \\
\hline$x, x_{\mathrm{i}}$ & $\begin{array}{l}\text { State-space formulation states, generic i-th state; or } \\
\text { generic local } x \text {-coordinate }\end{array}$ \\
\hline$x_{e}$ & Element local $x$-coordinates \\
\hline$X$ & $\begin{array}{l}\text { Global or substructure coordinate; or } \\
\text { state-space state equation(s) }\end{array}$ \\
\hline$X_{E}$ & Member end node $X$-coordinate in global coordinate system \\
\hline$X_{S}$ & Member start node $X$-coordinate in global coordinate system \\
\hline$X_{I N i}$ & $X$-coordinate in global coordinate system of the generic interface node \\
\hline$X_{S S}$ & Global or substructure coordinate \\
\hline$X_{T P}$ & TP reference point $X$-coordinate in global coordinate system \\
\hline$y, y_{i}$ & $\begin{array}{l}\text { State-space formulation outputs, generic i-th output; or } \\
\text { generic } y \text {-coordinate }\end{array}$ \\
\hline$y_{e}$ & Element local $y$-coordinates \\
\hline$Y$ & $\begin{array}{l}\text { Global or substructure coordinates; or } \\
\text { state-space output equation(s) }\end{array}$ \\
\hline$Y_{E}$ & Member end node $Y$-coordinate in global coordinate system \\
\hline$Y_{I N i}$ & $Y$-coordinate in global coordinate system of the generic interface node \\
\hline$Y_{S}$ & Member start node $Y$-coordinate in global coordinate system \\
\hline$Y_{S S}$ & Global or substructure coordinates \\
\hline$Y_{T P}$ & TP reference point $Y$-coordinate in global coordinat \\
\hline$Y_{1}$ & State-space output equation for ElastoDyn \\
\hline$Y_{2}$ & State-space output equation for HydroDyn \\
\hline
\end{tabular}




$\begin{array}{ll}z_{e} & \text { Element local } z \text {-coordinate } \\ Z & \text { Global or substructure coordinate } \\ Z_{E} & \text { Member end node } Z \text {-coordinate in global coordinate system } \\ Z_{I N i} & Z \text {-coordinate in global coordinate system of the generic interface node } \\ Z_{S} & \text { Member start node } Z \text {-coordinate in global coordinate system } \\ Z_{S S} & \text { Global or substructure coordinate } \\ Z_{T P} & \text { TP reference point } Z \text {-coordinate in global coordinate system }\end{array}$




\section{Table of Contents}

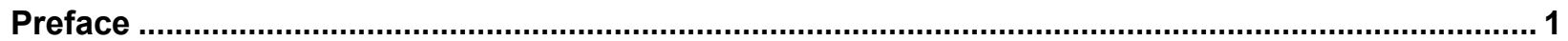

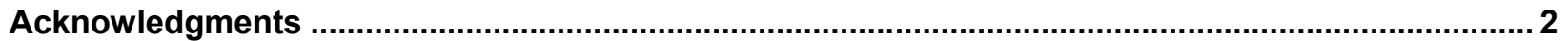

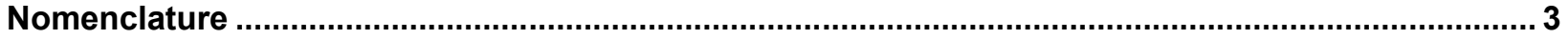

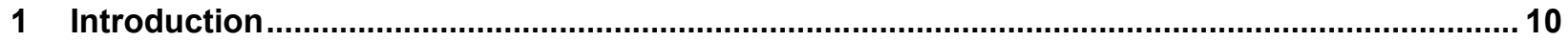

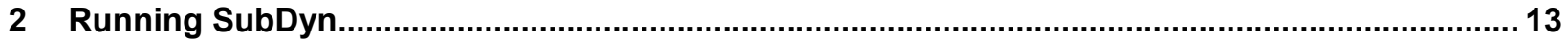

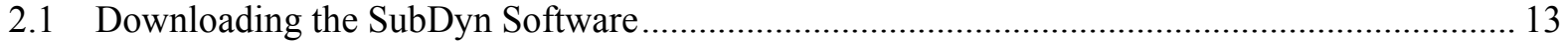

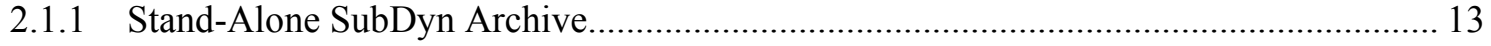

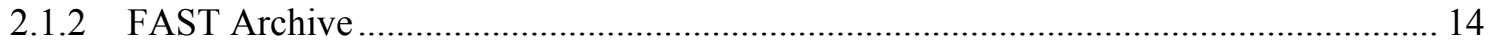

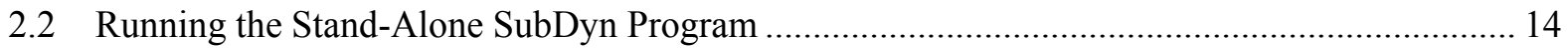

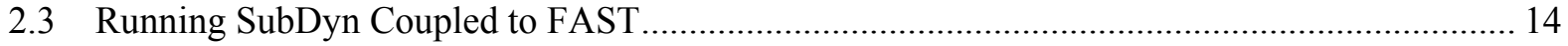

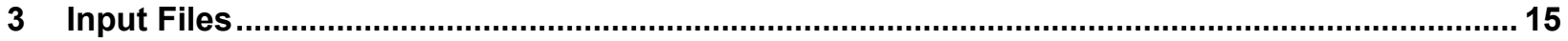

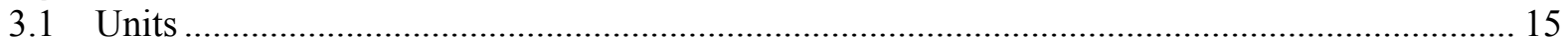

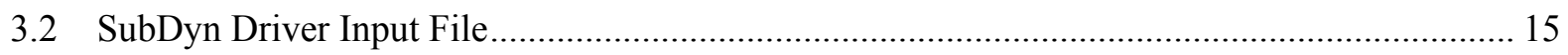

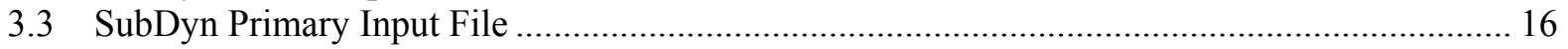

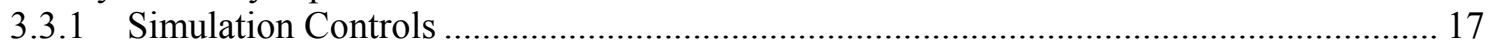

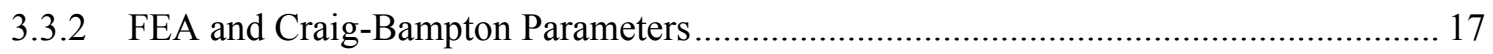

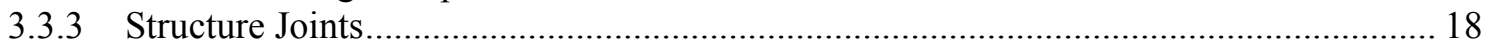

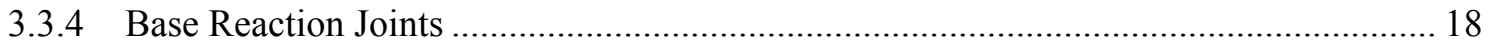

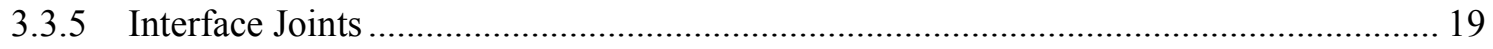

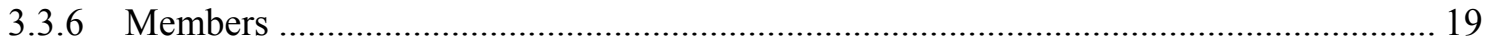

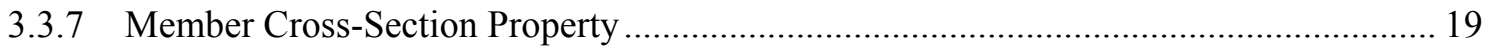

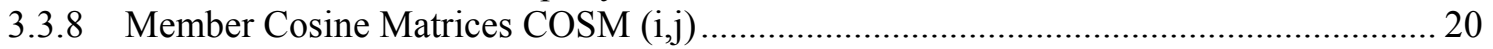

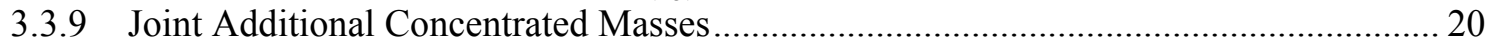

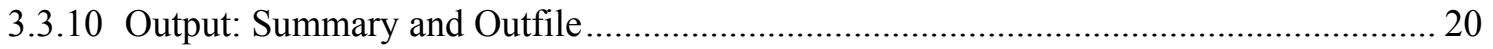

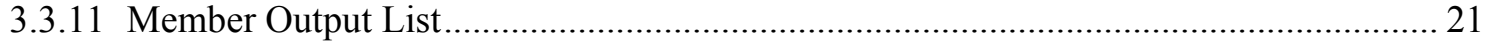

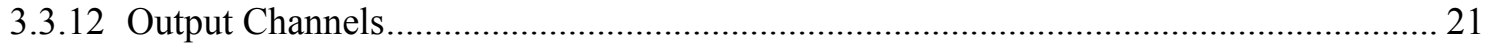

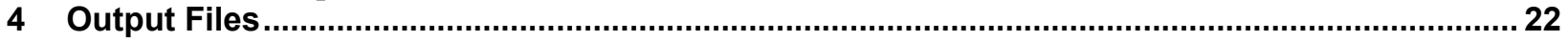

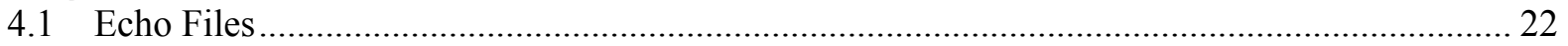

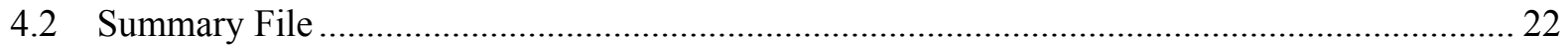

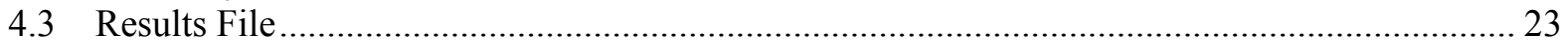

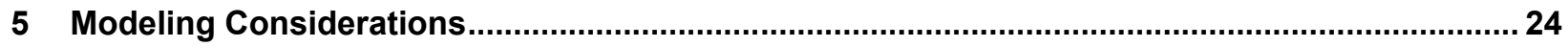

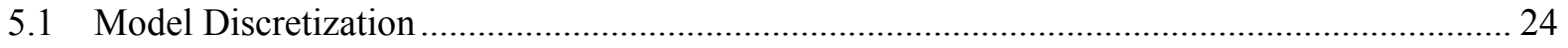

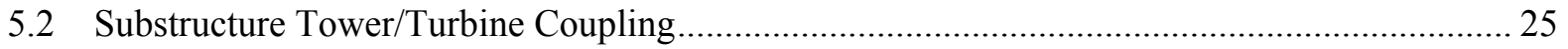

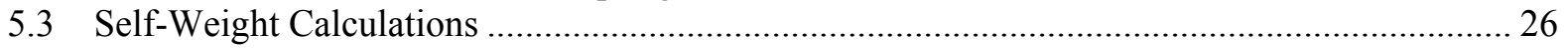

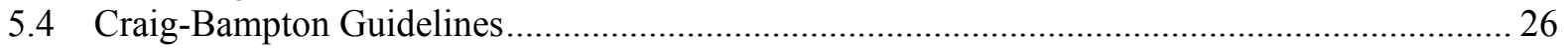

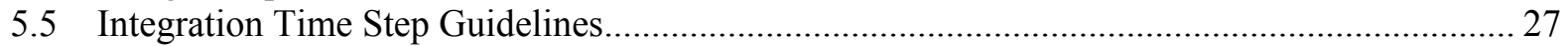

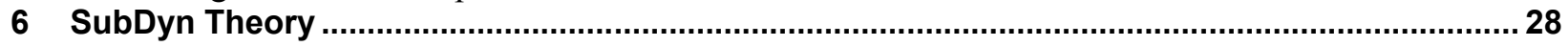

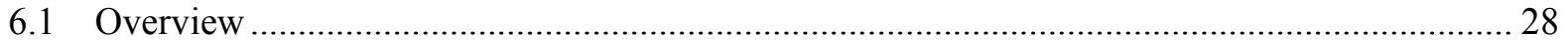

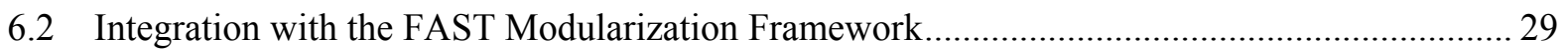

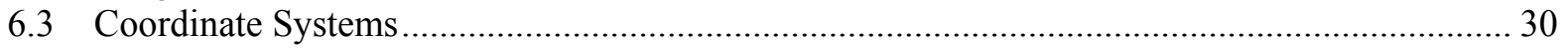

6.3.1 Global and Substructure Coordinate System: $(X, Y, Z)$ or $\left(X_{S S}, Y_{S S}, Z_{S S}\right) \ldots \ldots \ldots \ldots \ldots \ldots . \ldots \ldots$

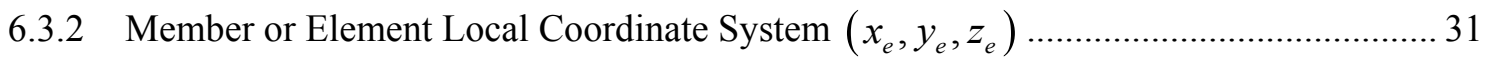

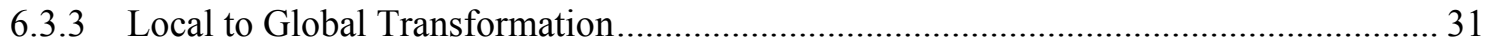

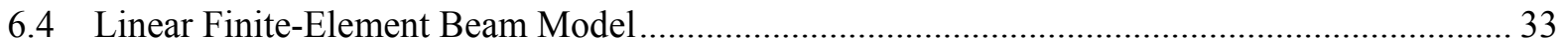

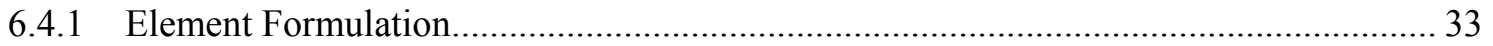

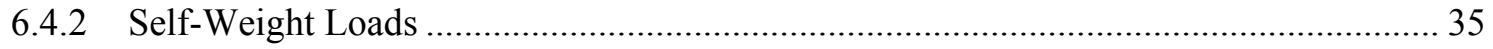

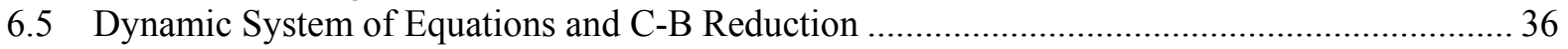

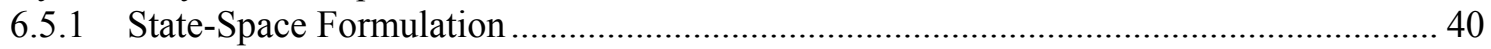

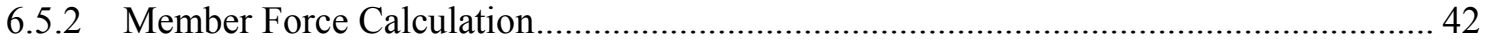




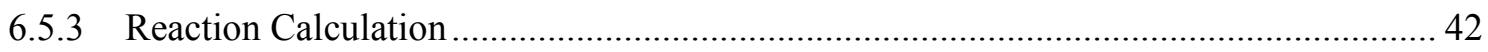

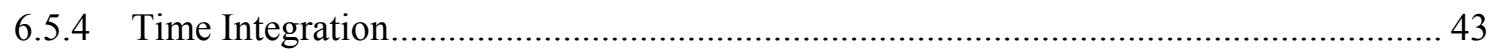

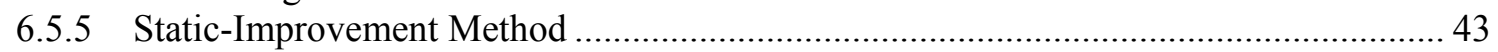

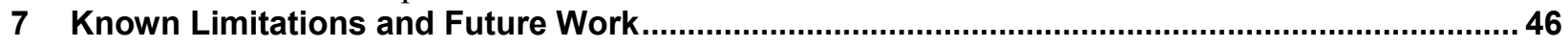

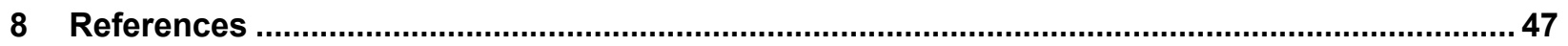

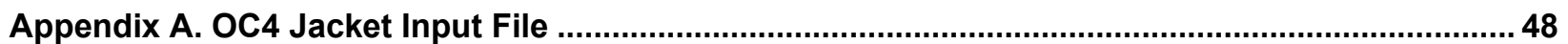

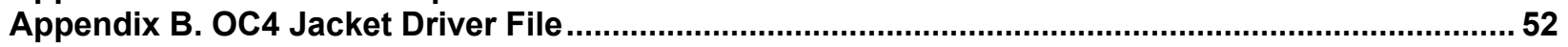

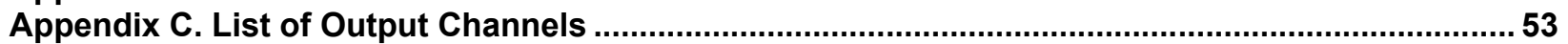

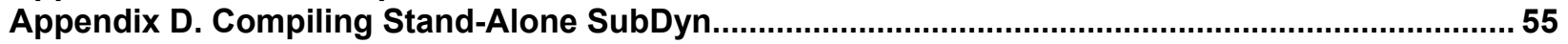

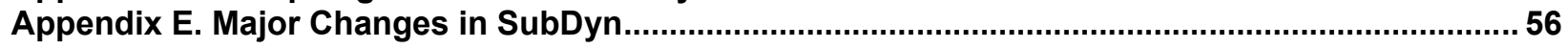

\section{List of Figures}

Figure 1. SubDyn layout within the modularization framework................................................... 29 Figure 2. Global (coincident with the substructure) coordinate system. Also shown are the DOFs associated with the TP reference point................................................................................... 30

\section{List of Tables}

Table 1. TP Reference Point Inputs Time-Series Data File Contents .............................................. 16

Table C-1. Possible SubDyn Output Parameters 


\section{Introduction}

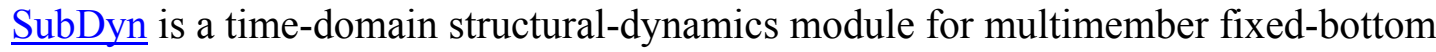
substructures created by the National Renewable Energy Laboratory (NREL) through U.S. Department of Energy Wind and Water Power Program support. The module has been coupled into the FAST aero-hydro-servo-elastic computer-aided engineering (CAE) tool. Substructure types supported by SubDyn include monopiles, tripods, jackets, and other lattice-type substructures common for offshore wind installations in shallow and transitional water depths. SubDyn can also be used to model lattice support structures for land-based wind turbines.

SubDyn follows the requirements of the FAST modularization framework, couples to FAST version 8, and provides new capabilities (relative to prior versions) for modeling the substructure dynamic loading on multimember substructures. (Refer to Appendix E and the changelog.txt file that is provided in the archives for more details about changes among different versions.) Uncoupled from FAST and in the absence of external loading other than gravity and interface motion, SubDyn can also be driven as a standalone code to compute the mode shapes, natural frequencies, and time-domain responses of substructures.

SubDyn relies on two main engineering schematizations: (1) a linear frame finite-element beam model (LFEB), and (2) a dynamics system reduction via the Craig-Bampton(C-B) method, together with a static-improvement method (SIM), greatly reducing the number of modes needed to obtain an accurate solution. More details can be found in Section 5, and in Song et al. (2013), Damiani et al. (2013), and Damiani and Song (2013).

In SubDyn, the substructure is considered to be clamped at the seabed and rigidly connected to the transition piece (TP) at the substructure top nodes (interface nodes). Other restraint formulations will be implemented in the future. Only the substructure structural dynamics are intended to be modeled within SubDyn. When integrated with FAST, the structural dynamics of the TP, tower, and rotor-nacelle assembly (RNA) are modeled within FAST's ElastoDyn module. (For full lattice support structures or other structures with no transition piece, however, the entire support structure up to the yaw bearing may be modeled within SubDyn.)

Loads and structural responses are transferred between SubDyn, HydroDyn, and ElastoDyn via the FAST driver program (glue code) to enable hydro-elastic interaction at each coupling time step. At the interface nodes, the TP six degree-of-freedom (DOF) displacements (three translations and three rotations), velocities, and accelerations are inputs to SubDyn from ElastoDyn; and the six reaction loads at the TP (three forces and three moments) are outputs from SubDyn to ElastoDyn. SubDyn also outputs the local substructure displacements, velocities, and accelerations to HydroDyn in order to calculate the local hydrodynamic loads that become inputs for SubDyn. In addition, SubDyn can calculate member internal reaction loads, as requested by the user.

The input file defines the substructure geometry, material properties, restraints, finite-element resolution, number of retained modes in the dynamics system reduction, modal damping coefficients, and auxiliary parameters. The geometry is defined by joint coordinates in the global reference system (inertial-frame coordinate system), with the origin at the intersection of the undeflected tower centerline with mean sea level (MSL) or ground level for land-based 
structures. A member connects two joints; multiple members may use a common joint. Nodes are the resultant of the member refinement into multiple (NDiv input parameter) elements (nodes are located at the ends of each element), and they are determined by the module. In the current release, member properties are outer diameter and wall thickness of tubular segments, whereas material properties are Young's modulus, shear modulus, and mass density. Member properties are specified at the joints; if properties change from one joint to the other, they will be linearly interpolated for the inner finite elements. Thus a tapered member will be treated as a cylindrical member with step-wise variation of its properties; in a future release, a tapered finite-element formulation will be implemented, and a more accurate representation of a tapered member will become available.

The hydrodynamic loads (including buoyancy) are computed by HydroDyn and transferred to SubDyn by the glue code at the submerged nodes. Additionally the self-weight distributed load components (from gravity) are calculated by SubDyn and applied at all the nodes. Note that other load and inertial properties may be input via the HydroDyn module input file, where marine growth and flooding/ballasting of the members can be specified.

This document is organized as follows. Section 1 details how to obtain the SubDyn and FAST software archives and run both the stand-alone SubDyn or SubDyn coupled to FAST. Section 2 describes the SubDyn input files. Section 3 discusses the output files generated by SubDyn; these include echo files, a summary file, and the results file. Section 4 provides modeling guidance when using SubDyn. The SubDyn theory is covered in Section 5. Section 6 outlines future work, and Section 7 contains a list of references. Example input files are shown in Appendixes A and B. A summary of available output channels are found in Appendix C. Instructions for compiling the stand-alone SubDyn program are detailed in Appendix D. Appendix E tracks the major changes we have made to SubDyn for each public release. 


\section{Running SubDyn}

This section discusses how to obtain and execute SubDyn from a personal computer. In what follows, both the stand-alone version and the FAST-coupled version of the software are considered.

\subsection{Downloading the SubDyn Software}

There are two renditions of the SubDyn software to choose from: stand-alone and fully coupled to the FAST simulator. Although the user may not necessarily need both renditions, he/she would likely need to be familiar with and run the stand-alone model when building a model of the substructure from scratch. The stand-alone version is also helpful for model troubleshooting and may benefit users who are interested in conducting aero-hydro-servo-elastic simulations of an offshore wind turbine. For this reason, SubDyn can be obtained from two different repositories: one for the stand-alone SubDyn and one for the fully coupled FAST simulator.

\subsubsection{Stand-Alone SubDyn Archive}

Users can download the stand-alone SubDyn archive from our Web server at http://wind.nrel.gov/designcodes/simulators/SubDyn. The file has a name similar to $S D \_$v1.01.00a-rrd.exe, but may have a different version number. The user can then download the self-extracting archive (.exe) to expand the archive into a folder he/she specifies.

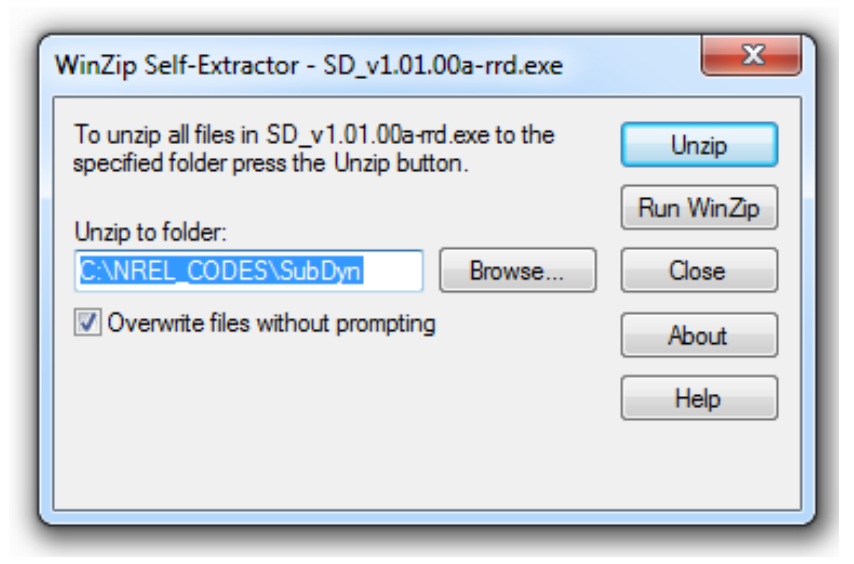

Figure 1. WinZip Self-Extractor main window

The archive contains the bin, CertTest, Compiling, Documentation, and Source folders. The bin folder includes the main executable file, SubDyn_win32.exe, which is used to execute the standalone SubDyn program. The CertTest folder contains a collection of sample SubDyn input files and driver input files that can be used as templates for the user's own models. If the user runs the CertTest.bat DOS script, he/she can compare the results to the ones stored in the NREL_Results subfolder. This manual may be found in the Documentation folder. The Compiling folder contains files for compiling the stand-alone SubDyn_win32.exe file with either Visual Studio or gFortran. The Fortran source code is located in the Source folder. 


\subsubsection{FAST Archive}

Download the FAST archive, which includes a coupling to SubDyn, from our Web server at http://wind.nrel.gov/designcodes/simulators/fast8/. The file has a name similar to FAST_v8.08.00.exe, but may have a different version number. Run the downloaded selfextracting archive (.exe) to expand the archive into a user-specified folder. The FAST executable file is located in the archive's bin folder. Example models using the NREL 5-MW reference turbine and various substructures are located in the CertTest folder. These include Test19: OC3Monopile, Test20: OC3-Tripod, Test21: OC4-jacket.

\subsection{Running the Stand-Alone SubDyn Program}

The stand-alone SubDyn program, SubDyn_win32.exe, simulates substructure dynamic responses of the user's input model, without coupling to FAST. Unlike the coupled version, the stand-alone software requires the use of a driver file in addition to the primary SubDyn input file. This driver file specifies initialization inputs normally provided to SubDyn by FAST, including motions of the TP reference point. Both the SubDyn summary file and the results output file are available when using the stand-alone SubDyn (see Section 3 for more information regarding the SubDyn output files).

Run the standalone SubDyn software from a DOS command prompt by typing, for example:

$>$ SubDyn_win32.exe MyDriverFile.dvr

where, MyDriverFile.dvr is the name of the SubDyn driver file, as described in Section 2.2. The SubDyn primary input file is described in Section 2.3.

\subsection{Running SubDyn Coupled to FAST}

Run the coupled FAST software from a DOS command prompt by typing, for example:

>FAST_win32.exe Test21.fst

where Test21.fst is the name of the primary FAST input file. This input file has a control flag to turn the SubDyn capabilities on or off within FAST, and a corresponding reference to the SubDyn input file. See the documentation supplied with FAST for further information. 


\section{Input Files}

The user configures the substructure model parameters, including its geometry and properties, via a primary SubDyn input file. When used in stand-alone mode, an additional driver input file is required. This driver file specifies initialization inputs normally provided to SubDyn by FAST, including motions of the TP reference point.

No lines should be added or removed from the input files, except in tables where the number of rows is specified.

\subsection{Units}

SubDyn uses the SI system (kg, m, s, N). Angles are assumed to be in radians unless otherwise specified.

\subsection{SubDyn Driver Input File}

The driver input file is only needed for the stand-alone version of SubDyn and contains inputs that are normally generated by FAST, and that are necessary to control the simulation for uncoupled models. It is possible to provide per-time-step inputs to SubDyn even in stand-alone mode, by tying the driver file to an additional input file containing time-histories of the TP input variables (displacements, velocities, and accelerations). A sample SubDyn driver input file is given in Appendix B.

Users can set the Echo flag in this file to TRUE so that SubDyn_win32.exe echoes the contents of the driver input file (useful for debugging errors in the driver file). The echo file has the naming convention of OutRootName.dvr.ech.OutRootName is specified in the SUBDYN section of the driver input file (see below).

Set the gravity constant using the Gravity parameter. SubDyn expects a magnitude, so in SI units this would be set to $9.80665 \frac{\mathrm{m}}{\mathrm{s}^{2}}$. WtrDpth specifies the water depth (depth of the seabed), based on the reference MSL, and must be a value greater than zero.

SDInputFile is the file name of the primary SubDyn input file. This name should be in quotations and can contain an absolute path or a relative path. All SubDyn-generated output files will be prefixed with OutRootName. If this parameter includes a file path, the output will be generated in that folder. NSteps specifies the number of simulation time steps, and TimeInterval specifies the time between steps. Next, set the coordinates in the global reference system of the

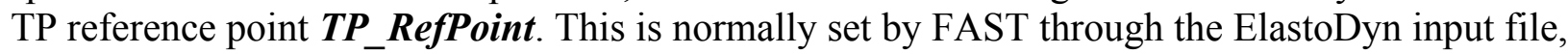
and it is the so-called platform reference point location. When coupled to FAST, the platform reference point location is identified by only one $(Z)$ coordinate. The interface joints, defined in SubDyn's main input file, are rigidly connected to this reference point. To utilize the same geometry definition within SubDyn's main input file, while still allowing for different substructure orientations, set $\boldsymbol{S u b R o t a t e} Z$ to a prescribed angle in degrees with respect to the global $Z$-axis. The entire substructure will be rotated by that angle. (This feature is only available in stand-alone mode.)

Setting InputsMod $=0$ forces all TP reference-point input motions to zero for all time steps. If the user sets InputsMod = 1, then he/she must set the steady (fixed) inputs in the STEADY 


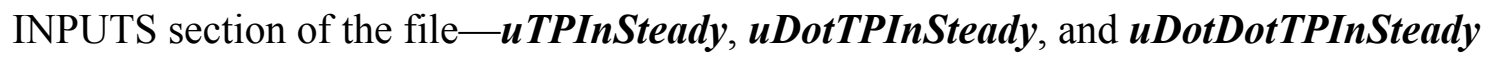
following the same convention as Table 1 (without time). Setting InputsMod=2, requires the time-series input file whose name is specified via the InputsFile parameter. The time-series input file is a text-formatted file. This file has no header lines, but NSteps rows, and each $i^{\text {th }}$ row has the first column showing time as $t=(i-1) *$ TimeInterval (the data will not be interpolated to other times). The remainder of each row is made of white-space-separated columns of floating point values representing the necessary motion inputs as shown in Table 1. All motions are specified in the global inertial-frame coordinate system. SubDyn does not check for physical consistency between motions specified for the TP reference point in the driver file.

Table 1. TP Reference Point Inputs Time-Series Data File Contents

\begin{tabular}{|c|c|c|}
\hline Column Number & Input & Units \\
\hline 1 & Time step value & $S$ \\
\hline $2-4$ & $\begin{array}{l}\text { TP reference point translational } \\
\text { displacements along } X, Y \text {, and } Z\end{array}$ & $m$ \\
\hline $5-7$ & $\begin{array}{c}\text { TP reference point rotational } \\
\text { displacements about } X, Y \text {, and } Z \\
\text { (small angle assumptions apply) }\end{array}$ & radians \\
\hline $8-10$ & $\begin{array}{l}\text { TP reference point translational } \\
\text { velocities along } X, Y \text {, and } Z\end{array}$ & $\frac{m}{s}$ \\
\hline $11-13$ & $\begin{array}{l}\text { TP reference point rotational } \\
\text { velocities about } X, Y \text {, and } Z\end{array}$ & $\frac{\text { radians }}{s}$ \\
\hline $14-16$ & $\begin{array}{l}\text { TP reference point translational } \\
\text { accelerations along } X, Y, \text { and } Z\end{array}$ & $\frac{m}{s^{2}}$ \\
\hline $17-19$ & $\begin{array}{l}\text { TP reference point rotational } \\
\text { accelerations about } X, Y \text {, and } Z\end{array}$ & $\frac{\text { radians }}{s^{2}}$ \\
\hline
\end{tabular}

\subsection{SubDyn Primary Input File}

The SubDyn input file defines the substructure geometry, integration and simulation options, finite-element parameters, and output channels. The geometry of members is defined by joint coordinates of the undisplaced substructure in the global reference system (inertial-frame coordinate system), with the origin at the intersection of the undeflected tower centerline with MSL or ground level for land-based structures. A member connects two joints; multiple members can use a common joint. The hydrodynamic and gravity loads are applied at the nodes, which are the resultant of member refinement into multiple (NDiv input) elements (nodes are located at the ends of each element), and they are calculated by the module. Member properties, which include outer diameter, thickness, material density, and Young's and shear moduli are specified at the joints; if properties change from one joint to the other, they will be linearly interpolated for the inner nodes. Unlike the geometric properties, the material properties are not allowed to change within a single member. 
Future releases will allow for members of different cross-sections, i.e., noncircular members. For this reason, the input file has (currently unused) sections dedicated to the identification of direction cosines that in the future will allow the module to identify the correct orientation of noncircular members. The current release only accepts tubular (circular) members.

The file is organized into several functional sections. Each section corresponds to an aspect of the SubDyn model and substructure.

If this manual refers to an ID in a table entry, it is an integer identifier for the table entry and must be unique for a given table entry.

A sample SubDyn primary input file is given in Appendix A.

The input file begins with two lines of header information, which is for the user but is not used by the software.

\subsubsection{Simulation Controls}

Set the Echo flag to TRUE so that SubDyn echoes the contents of the SubDyn input file (useful for debugging errors in the input file). The echo file has the naming convention of

OutRootName.SD.ech. OutRootName is either specified in the SUBDYN section of the driver input file when running SubDyn standalone, or by FAST when running a coupled simulation.

SDdeltaT specifies the time step of the integration in seconds. The keyword 'DEFAULT' may be used to indicate that the module should employ the time step prescribed by the glue-code (FAST/driver program).

IntMethod specifies the integration algorithm to use. There are four options: 1) Runge-Kutta $4^{\text {th }}$ order explicit (RK4); 2) Adams-Bashforth $4^{\text {th }}$-order explicit predictor (AB4); 3) AdamsBashforth-Moulton $4^{\text {th }}$-order explicit predictor-corrector (ABM4); 4) Adams-Moulton implicit $2^{\text {nd }}$-order (AM2).

SttcSolve is a flag that specifies whether SIM (see Sections 4 and 5) shall be employed. Through this method, all (higher frequency) modes that are not considered by the C-B reduction are treated quasi-statically. This treatment helps minimize the number of retained modes to capture effects such as static gravity and buoyancy loads, and high-frequency loads transferred from the turbine.

\subsubsection{FEA and Craig-Bampton Parameters}

FEMMod specifies one of the following options for finite-element formulation: 1) EulerBernoulli; 3) Timoshenko. Tapered formulations (2 and 4) have yet to be implemented and will be available in a future release.

NDiv specifies the number of elements per member. Analysis nodes are located at the ends of elements and the number of analysis nodes per member equals NDiv + 1. NDiv is applied uniformly to all members regardless of the member's length, hence it could result in small elements in some members and long elements in other members. Increasing the number of 
elements per member may increase accuracy, with the trade-off of increased memory usage and computation time. We recommend using NDiv > 1 when modeling tapered members.

$\boldsymbol{C B M o d}$ is a flag that specifies whether or not the C-B reduction should be carried out by the module. If FALSE, then the full finite-element model is retained and Nmodes is ignored.

Nmodes sets the number of internal C-B modal DOFs to retain in the C-B reduction. Nmodes $=0$ corresponds to a Guyan (static) reduction; retaining all modes corresponds to keeping the full finite-element model and is virtually equivalent to $\boldsymbol{C B M o d}=$ FALSE. Nmodes is ignored if CBMod is set to FALSE.

JDampings specifies value(s) of damping coefficients as a percentage of critical damping for the retained C-B modes. If the number of JDampings is less than the retained modes, the last value will be replicated for all the remaining modes. For more information on these parameters and guidelines on how to set them, see Sections 4 and 5.

\subsubsection{Structure Joints}

The finite-element model is based on a substructure composed of joints interconnected by members. NJoints is the user-specified number of joints, and determines the number of rows in the subsequent table. Because a member connects two joints, NJoints must be greater than or equal to two. Each joint listed in the table is identified by a unique integer, JointID; each integer between one and NJoints must be present in the table, but they need not be sequential. The $(X, Y, Z)$ coordinate of each joint is specified in the substructure (SS) coordinate system, which coincides with the global inertial-frame coordinate system via JointXss, JointYss, and JointZss, respectively. This version of SubDyn cannot calculate joint overlap when multiple members meet at a common joint; future releases will enable joint overlap and node offset calculations.

Note for HydroDyn coupling: modeling a fixed-bottom substructure embedded into the seabed (e.g., through piles or suction buckets) requires that the lowest member joint(s) in HydroDyn lie(s) below the water depth. Placing a joint at or above the water depth will result in static and dynamic pressure loads being applied. When SubDyn is coupled to FAST, the joints and members need not match between HydroDyn and SubDyn-FAST's mesh-mapping utility handles transfer of motion and loads across meshes in a physically relevant manner (Sprague et al. 2014), but consistency between the joints and members in HydroDyn and SubDyn is advised.

\subsubsection{Base Reaction Joints}

SubDyn requires the user to specify the boundary joints. NReact should be set equal to the number of joints at the bottom of the structure (i.e., seabed) that are fully constrained; NReact also determines the number of rows in the subsequent table. Each joint listed in the table is identified by a unique integer, RJointID, which must correspond to the JointID value found in the STRUCTURE JOINTS table. The flags RctTDXss, RctTDYss, RctTDZss, RctRDXss, RctRDYss, RctRDZss indicate the values of fixities for the three translations (TD) and three rotations (RD) in the SS coordinate system (global inertial-frame coordinate system), with one denoting fixed and zero denoting free (instead of TRUE/FALSE). This version of SubDyn cannot handle partially restrained joints, so all flags must be set to one; future releases will allow for different degrees of fixity. 


\subsubsection{Interface Joints}

SubDyn requires the user to specify the boundary joints. NInterf should be set equal to the number of joints at the top of the structure (i.e., TP); NInterf also determines the number of rows in the subsequent table. Note that these joints will be assumed to be rigidly connected to the platform reference point of ElastoDyn (see FAST documentation) when coupled to FAST, or to the TP reference point if SubDyn is run in stand-alone mode. Each joint listed in the table is identified by a unique integer, IJointID, which must correspond to the JointID value found in the STRUCTURE JOINTS table. The flags ItfTDXss, ItfTDYss, ItfTDZss, ItfRDXss, ItfRDYss, ItfRDZss indicate the values of fixities for the three translations (TD) and three rotations (RD) in the SS coordinate system (global inertial-frame coordinate system), with one denoting fixed and zero denoting free (instead of TRUE/FALSE). This version of SubDyn cannot handle partially restrained joints, so all flags must be set to one; future releases will allow for different degrees of fixity.

\subsubsection{Members}

NMembers is the user-specified number of members and determines the number of rows in the subsequent table. Each member listed in the table is identified by a unique integer, MemberID; each integer between one and NMembers must be present in the table, but they need not be sequential. For each member distinguished by MemberID, MJointID1 specifies the starting joint and MJointID2 specifies the ending joint, corresponding to an identifier (JointID) from the STRUCTURE JOINTS table. Likewise, MPropSetID1 corresponds to the identifier (PropSetID from the MEMBER X-SECTION PROPERTY table (discussed subsequently) for starting crosssection properties and MPropSetID2 specifies the identifier for ending cross-section properties, allowing for tapered members. COSMID refers to the IDs of the members' cosine matrices; the current release ignores this column.

\subsubsection{Member Cross-Section Property}

Members in SubDyn are assumed to be straight, circular, possibly tapered, and hollow cylinders. Future releases will allow for generic cross-sections to be employed. These special cross-section members will be defined in the second of two tables in the input file, which is currently ignored.

For the circular cross-section members, properties needed by SubDyn are material Young's modulus, YoungE, shear modulus, ShearG, and density, MatDens, member outer diameter, $\boldsymbol{X s e c D}$, and member thickness, XsecT. Users will need to create an entry in the first table within this section of the input file distinguished by PropSetID, for each unique combination of these five properties. The member property-set table contains NPropSets rows. The member property sets are referred to by their PropSetID in the MEMBERS table, as described in Section 2.3.6. Note, however, that although diameter and thickness can change within an individual member, SubDyn will not allow material properties to change within an individual member.

The second table in this section of the input file (not to be used in this release) will have NXPropSets rows (assumed to be zero for this release), and have additional entries when compared to the previous table, including: cross-sectional area (XsecA); cross-sectional shear area along $x$ and $y$ (local principal axes) (XsecAsx, Xsec Asy); cross-sectional area second moment of inertia about $x$ and $y$ (XsecJxx, XsecJyy); and cross-sectional area polar moment of 
inertia (XsecJ0). The member cosine matrix section (see Section 2.3.8) will help determine the correct orientation of the members within the assembly.

\subsubsection{Member Cosine Matrices COSM (i,j)}

This table is not currently used by SubDyn, but in future releases it will need to be populated if members with cross-sections other than circular will be employed.

NCOSMS rows, one for each unique member orientation set, will need to be provided. Each row of the table will list the nine entries of the direction cosine matrices (COSM11, COSM12,...COSM33, for matrix elements $(1,1),(1,2), \ldots(3,3))$ that establish the orientation of the local member axes ( $x, y$ principal axes in the cross-sectional plane, $z$ along the member longitudinal axis) with respect to the SS coordinate system (local-to-global transformation matrices).

\subsubsection{Joint Additional Concentrated Masses}

SubDyn can accept $\mathbf{N C m a s s}$ lumped masses/inertias defined at the joints. The subsequent table will have NCmass rows, in which for each joint distinguished by CMJointID (corresponding to an identifier, JointID, from the STRUCTURE JOINTS table), JMass specifies the lumped mass value, and $\boldsymbol{J M X X} \boldsymbol{J} \boldsymbol{M} \boldsymbol{Y} \boldsymbol{Y}, \boldsymbol{J M Z Z}$ specify the mass second moments of inertia with respect to the SS coordinate system.

\subsubsection{Output: Summary and Outfile}

In this section of the input file, the user sets flags and switches for the desired output behavior.

Specifying $\boldsymbol{S D S U m}=$ TRUE causes SubDyn to generate a summary file with name OutRootname.SD.sum. OutRootName is either specified in the SUBDYN section of the driver input file when running SubDyn in stand-alone mode, or by the FAST program when running a coupled simulation. See Section 3.2 for summary file details.

In this release, $\boldsymbol{O u t C O S M}$ is ignored. In future releases, specifying $\boldsymbol{O u t C O S M}=$ TRUE will cause SubDyn to include direction cosine matrices (undeflected) in the summary file for only those members requested in the list of output channels.

Specifying OutAll = TRUE causes SubDyn to output forces and moments at all of the joints, (not internal nodes). That is, the static (elastic) and dynamic (inertia) components of the three forces and three moments at the end node of each member connected to a given joint are output for all joints. These outputs are included within the OutRootName.SD.out output file in addition to those directly specified through the output channels section below.

If OutSwtch is set to one, outputs are sent to a file with the name OutRootName.SD.out. If OutSwtch is set to two, outputs are sent to the calling program (FAST) for writing. If OutSwtch is set to three, both file outputs occur. In stand-alone mode, setting OutSwtch to two results in no output file being produced.

If TabDelim is set to TRUE and OutSwtch is set to one, the output file OutRootName.SD.out will be tab-delimited. 
With OutDec set to an integer value greater than one, the output file data rate will be decimated, and only every OutDec-th value will be written to the file.

The OutFmt and OutSFmt parameters control the formatting for the output data and the channel headers, respectively. SubDyn currently does not check the validity of these format strings. They need to be valid Fortran format strings. OutSFmt is used for the column header and OutFmt is used for the channel data. Therefore, in order for the headers and channel data to align properly, the width specification should match. For example:

"ES11.4" OutFmt

"A11" OutSFmt

\subsubsection{Member Output List}

SubDyn can output load and kinematic quantities at up to nine locations along up to nine different members, for a total of 81 possible local member output locations. NMOutputs specifies the number of members. The user must create a table entry for each requested member. Within a table entry, MemberID is the ID specified in the MEMBERS table, and NOutCnt specifies how many nodes along the member will be available for output. NodeCnt specifies those node numbers as an integer index from the start-joint (node 1) to the end-joint (node NDiv +1 ) of the member. The outputs specified in the OUTPUT CHANNELS section determines which quantities are actually output at these locations.

\subsubsection{Output Channels}

This section controls output quantities generated by SubDyn. Enter one or more lines containing quoted strings that in turn contain one or more output parameter names. Separate output parameter names by any combination of commas, semicolons, spaces, and/or tabs. If a parameter name is prefixed with a minus sign, "-", underscore, "_,", or the characters "m" or "M", SubDyn will multiply the value for that channel by -1 before writing the data. The parameters are written in the order they are listed in the input file. SubDyn allows the use of multiple lines so that users can break their lists into meaningful groups and so the lines can be shorter. Comments may also be entered after the closing quote on any of the lines. Entering a line with the string "END" at the beginning of the line or at the beginning of a quoted string found at the beginning of the line will cause SubDyn to quit scanning for more lines of channel names. Modal kinematics and membernode-, base-, and interface-related kinematic and load quantities can be selected. Member-noderelated data follow the organization described in Section 2.3.11. If SubDyn encounters an unknown/invalid channel name, it prints an error message and halts execution. Please refer to Appendix $\mathrm{C}$ for a complete list of possible output parameters. 


\section{Output Files}

SubDyn produces three types of output files: an echo file, a summary file, and a time-series results file. The following sections detail the purpose and contents of these files.

\subsection{Echo Files}

If the user sets the Echo flag to TRUE in the SubDyn driver file or the primary SubDyn input file, the contents of those files will be echoed to a file with the naming conventions,

OutRootName.dvr.ech for the driver input file and OutRootName.SD.ech for the primary SubDyn input file. OutRootName is either specified in the SUBDYN section of the driver input file, or by the FAST program. The echo files are helpful for debugging the input files. The contents of an echo file will be truncated if SubDyn encounters an error while parsing an input file. The error usually corresponds to the line after the last successfully echoed line.

\subsection{Summary File}

SubDyn generates a summary file with the naming convention, OutRootName.SD.sum if the SDSum parameter is set to TRUE. This file summarizes key information about the substructure model, including:

- Undisplaced node geometry. A table lists all of the (NNodes) nodes and the $X, Y, Z$ coordinates in the global SS coordinate system. Note that NNodes may be greater or equal to NJoints, depending on NDiv (primary input file parameters).

- Element connectivity and properties at end nodes. A table lists all (NElems) elements, the start and end nodes (Node_I, Node_J) and the ID of the property set (Prop_I,Prop_J) at the start and end nodes. NElems may be greater or equal to NMembers, depending on NDiv (primary input file parameters).

- Property sets. If tapered members are used, additional property sets may be included beyond those specified in the main input file. Headers and their meanings are identical to those described in Section 2.3.7.

- Reaction DOFs (fixity) and interface DOFs (fixity). The actual indices of the DOFs $\left(\boldsymbol{D O F} \boldsymbol{F}_{-} \boldsymbol{I D}\right)$ associated with reaction and interface nodes are listed together with the $(1 / 0)$ flag to distinguish the fixity level.

- Concentrated mass schedule. This is an echo of the equivalent section in the primary input file. Refer to Section 2.3.9.

- Member schedule including connectivity to joints, nodes, and their masses. A table lists all of the members by identifier (MemberID), with their start and end nodes (Joint1_ID, Joint2_ID), associated mass (Mass), and list of node identifiers along the length of the members.

- Direction cosine matrices for the members. Each row (columns 2-10) corresponds to the direction cosine matrix entries $(\boldsymbol{D C}(1,1)$ through $\boldsymbol{D C}(3,3))$ for the member whose 
identifier is listed in the first column. The direction cosine matrices specify the transformation from the global reference to the local coordinate system for each member.

- Sorted eigenfrequencies (in Hertz [Hz]) for the full substructural system (neglecting a possible coupling to ElastoDyn through FAST), assuming the TP reference point is a free end. There are a total of NDOFs eigenfrequencies and eigenvectors.

- Sorted eigenfrequencies (in $\mathrm{Hz}$ ) for the C-B reduced system, assuming the TP reference point is a fixed end. There are a total of Nmodes $\mathrm{C}-\mathrm{B}$ reduced eigenfrequencies and eigenvectors.

- Full substructural system eigenvectors. Each column represents an eigenvector associated with the corresponding eigenfrequency identified previously in the file.

- C-B reduced system eigenvectors (PhiM matrix). Each column represents an eigenvector associated with the corresponding eigenfrequency identified previously in the file.

- PhiR matrix or displacements of the internal nodes caused by unit rigid body motions of the interface DOFs (see Section 5). Each column of the matrix represents the internal DOF displacements for a given unit rigid-body motion along an interface DOF for each base and interface joint.

- Substructure equivalent stiffness and mass matrices referred to the TP reference point $(\boldsymbol{K B B t}$ and $\boldsymbol{M B B t})$, based on a Guyan reduction. These are useful to calculate effects of substructure flexibility while calculating tower eigenmodes for ElastoDyn.

- Rigid-body-equivalent mass matrix relative to global origin (MRB); a 6x6 mass matrix.

- Substructure total (dry) mass.

- Substructure center of mass coordinates in the global coordinate system.

The various sections of the summary file and variables are self-explanatory and easily identifiable in the file.

\subsection{Results File}

The SubDyn time-series results are written to a text-based file with the naming convention OutRootName.SD.out when OutSwtch is set to either one or three. If SubDyn is coupled to FAST and OutSwtch is set to two or three, then FAST will generate a master results file that includes the SubDyn results. The results are in table format, where each column is a data channel (the first column always being the simulation time), and each row corresponds to a simulation time step. The data channels are specified in the OUTPUT CHANNELS section of the input file. The column format of the SubDyn-generated file is specified using the OutFmt and OutSFmt parameters of the input file. 


\section{Modeling Considerations}

SubDyn was designed as a flexible tool for modeling a wide range of substructures for both landbased and offshore applications. This section provides some general guidance to help construct models that are compatible with SubDyn.

Please refer to the theory in Section 8 for detailed information about SubDyn's coordinate systems, and the implementation approach we have followed in SubDyn.

\subsection{Model Discretization}

SubDyn allows for the specification of arbitrary multimember structure geometries. The user defines the geometry of a structure in SubDyn using joints and members; in particular, the user specifies a list of joints that represent the endpoints of beams, and the connectivity between one or more members at each joint. Members and their cross-sectional properties are then defined between two joints. Members can be further subdivided into multiple (NDiv) subelements to increase the model resolution; nodes, where the numerical calculations take place, are located at the endpoints of each element. To keep the mesh as uniform as possible when using NDiv, the initial member definition should also have rendered a uniform mesh. For tapered members, we recommend setting $N$ Div $>1$.

Improper discretization of the members may decrease the accuracy of the model. When SubDyn is coupled to FAST, the joints and members need not match between HydroDyn and SubDynFAST's mesh-mapping utility handles transfer of motion and loads across meshes in a physically relevant manner (Sprague et al. 2014), but consistency between the joints and members in HydroDyn and SubDyn is advised.

For offshore applications, because of the exponential decay of hydrodynamic loads with depth, HydroDyn requires higher resolution near the water free surface to properly capture loads as waves oscillate about the still water level (SWL). We recommend, for instance, that the HydroDyn discretization not exceed element lengths of $0.5 \mathrm{~m}$ in the region of the free surface ( 5 to $10 \mathrm{~m}$ above and below SWL), $1.0 \mathrm{~m}$ between $25-$ and $50-\mathrm{m}$ depth, and $2.0 \mathrm{~m}$ in deeper waters. When SubDyn is coupled to HydroDyn through FAST for the analysis of fixed-bottom offshore systems, we recommend that the length ratio between elements of SubDyn and HydroDyn not exceed 10 to 1 . As such, we recommend that the SubDyn discretization not exceed element lengths of $5 \mathrm{~m}$ in the region of the free surface, $10 \mathrm{~m}$ down to $25-$ to $50-\mathrm{m}$ depth, and $20 \mathrm{~m}$ in deeper waters. These are not absolute rules, but rather a good starting point that will likely require refinement for a given substructure. Additional considerations for SubDyn discretization include aspects that will impact structural accuracy, such as member weight, substructure modes and/or natural frequencies, load transfer, tapered members, and so on.

Members in SubDyn are assumed to be straight circular (and possibly tapered) cylinders. Future releases will enable the use of generic cross-sectional shapes.

Note that in HydroDyn, all members that are embedded into the seabed (e.g., through piles or suction buckets) must have a joint that is located below the water depth. In SubDyn, the bottom joint will be considered clamped and therefore need not be located below seabed. For example, if the water depth is set to $20 \mathrm{~m}$, and the user is modeling a fixed-bottom monopile, then the 
bottom-most joint in SubDyn can be set at $Z=-20 \mathrm{~m}$; HydroDyn, however, needs to have a Zcoordinate such that $Z<-20 \mathrm{~m}$. This configuration avoids HydroDyn applying static pressure loads on the bottom of the structure. The user may still want to create nodes below the seabed to simulate a fixity length.

\subsection{Substructure Tower/Turbine Coupling}

In ElastoDyn, the 6 DOFs of the platform must be enabled to couple loads and displacements between the turbine and the substructure. The platform reference point coordinates in ElastoDyn should also be set equal to the TP reference point (normally the tower base flange location or TP centroid or center of mass) that the user may have set in the stand-alone mode for checking the SubDyn model. A rigid connection between the SubDyn interface joints and TP reference point (三 platform reference point) will be assumed.

ElastoDyn also needs tower mode shapes specified (coefficients of best-fit sixth-order polynomials). They can be derived with an appropriate software (finite-element analysis, energy methods, or analytically) and by making use of the SubDyn-derived equivalent substructure stiffness and mass matrices $\left(\boldsymbol{K B B} \boldsymbol{B} / \tilde{K}_{B B}\right.$ and $\boldsymbol{M B B t} / \tilde{M}_{B B}$ matrices found in the SubDyn summary file) for the boundary conditions at the base of the tower. For instance, using NREL's BModes software, the SubDyn-obtained matrices can be used in place of the hydrodynamic stiffness (hydro_K) and mass matrices (hydro_M) (mooring_K may be set to zero); by setting the $\boldsymbol{h u b}$ _conn boundary condition to two (free-free), BModes will calculate the mode shapes of the tower when tower cross-sectional properties are supplied. To obtain eigenmodes that are compatible with the FAST modal treatment of the tower (i.e., no axial or torsional modes, and no distributed rotational-inertia contribution to the eigenmodes), the tower distributed properties should be modified accordingly (e.g., by reducing mass moments of inertia towards zero, and by increasing torsional and axial stiffness, while assuring convergence of the results; see also https://wind.nrel.gov/forum/wind/viewtopic.php? $\mathrm{f}=4 \& \mathrm{t}=742$ ).

The rotational inertia of the undeflected tower about its centerline is not currently accounted for in ElastoDyn. Thus, when the nacelle-yaw DOF is enabled in ElastoDyn there will not be any rotational inertia of the platform-yaw DOF (which rotates the tower about its centerline) when both the platform-yaw inertia in ElastoDyn is zero and the tower is undeflected. To avoid a potential division-by-zero error in ElastoDyn when coupled to SubDyn, we recommend setting the platform-yaw inertia (Ptfm YIner) in ElastoDyn equal to the total rotational inertia of the undeflected tower about its centerline.

The C-B method assumes no damping for the interface modes. This is equivalent to having six undamped rigid-body DOFs at the TP reference point in the absence of aerodynamic or hydrodynamic damping. Experience has shown that negligible platform-heave damping can cause numerical problems when SubDyn is coupled to FAST. One way to overcome this problem is to augment overall system damping with an additional linear damping for the platform-heave DOF. This augmentation can be achieved quite easily by calculating the damping from Eq. 1 and specifying this as the $(3,3)$ element of HydroDyn's additional linear damping matrix, AddBLin. Experience has shown that a damping ratio of $1 \%$ of critical $(\zeta=0.01)$ is sufficient. In Eq. 1, $K_{33}^{(S D)}$ is the equivalent heave stiffness of the substructure (the $(3,3)$ element 
of the $\tilde{K}_{B B}(\boldsymbol{K B B} \boldsymbol{t})$ matrix found in the SubDyn summary file, see also Section 5), ${ }^{M_{33}^{(S D)}}$ is the equivalent heave mass of the substructure (the $(3,3)$ element of the $\tilde{M}_{B B}(\boldsymbol{M B B} \boldsymbol{t})$ matrix found in the SubDyn summary file, see also Section 5), and $M^{(E D)}$ is the total mass of the rotor, nacelle, tower, and TP (found in the ElastoDyn summary file).

$$
C_{33}^{(H D)}=2 \zeta \sqrt{K_{33}^{(S D)}\left(M_{33}^{(S D)}+M^{(E D)}\right)}
$$

To minimize extraneous excitation of the platform-heave DOF, it is useful to set the initial platform-heave displacement to its natural static-equilibrium position, which can be approximated by Eq. 2, where $g$ is the magnitude of gravity. PtfmHeave from Eq. 2 should be specified in the initial conditions section of the ElastoDyn input file.

$$
\text { PtfmHeave }=-\frac{\left(M_{33}^{(S D)}+M^{(E D)}\right) g}{K_{33}^{(S D)}}
$$

\subsection{Self-Weight Calculations}

SubDyn will calculate the self-weight of the members and apply appropriate forces and moments at the element nodes. Lumped masses will also be considered as concentrated gravity loads at prescribed joints. The array of self-weight forces can be seen in the summary file if the code is compiled with DEBUG compiler directives. In general, SubDyn assumes that structural motions of the substructure are small, such that 1) small-angle assumptions apply to structural rotations, 2) the so-called P- $\Delta$ effect is negligible, and therefore undeflected node locations are used for self-weight calculations.

When SubDyn is coupled to HydroDyn through FAST, the hydrodynamic loads, which include buoyancy, marine-growth weight, and wave and current loads, will be applied to the effective, deflected location of the nodes by the mesh-mapping routines in the glue code. Those loads, however, are based on wave kinematics at the undeflected position (see Jonkman et al. 2014 for more information).

\subsection{Craig-Bampton Guidelines}

When SubDyn is coupled with FAST, it is important to choose a sufficient number of C-B modes, ensuring that the physical modes of the coupled system are properly captured by the coupled model. We recommend that all physical modes up to at least 2-3 Hz be captured; wind, wave, and turbine excitations are important for frequencies up to 2-3 Hz. Eigen analysis of the linearized, coupled system will make checking this condition possible; however, the linearization process has yet to be implemented in FAST v8. Before then, experience has shown that it is sufficient to enable all C-B modes up to $10 \mathrm{~Hz}$. (The natural frequencies of the C-B modes are written to the SubDyn summary file.) If SIM is not enabled, in addition to capturing physical modes up to a given frequency, the highest C-B mode must include the substructure axial modes so that gravity loading from self-weight is properly accounted for within SubDyn. This inclusion likely requires enabling a high number of $\mathrm{C}-\mathrm{B}$ modes, reducing the benefit of the $\mathrm{C}-\mathrm{B}$ reduction. Thus, we recommend employing the C-B reduction with SIM enabled. Because of the fixed- 
fixed treatment of the substructure boundary conditions in the $\mathrm{C}-\mathrm{B}$ reduction, the $\mathrm{C}-\mathrm{B}$ modes will always have higher natural frequencies than the physical modes.

\subsection{Integration Time Step Guidelines}

Another consideration for creating SubDyn input files is the time step size. SubDyn offers three explicit time-integrators - the fourth-order Runge-Kutta (RK4), fourth-order Adams-Bashforth (AB4), fourth-order Adams-Bashforth-Moulton (ABM4) methods - and the implicit secondorder Adams-Moulton (AM2) method. Users have the option of using the global time step from the glue code or an alternative SubDyn-unique time step that is an integer multiple smaller than the glue-code time step. It is essential that a small enough time step is used to ensure solution accuracy (by providing a sufficient sampling rate to characterize all key frequencies of the system), numerical stability of the selected explicit time-integrator, and that the coupling with FAST is numerically stable.

For the RK4 and ABM4 methods, we recommend that the SubDyn time step follow the relationship shown in Eq. 3, where $f_{\max }$ is the higher of 1) the highest natural frequency of the retained C-B modes and 2) the highest natural frequency of the physical modes when coupled to FAST. Although the former can be obtained from the SubDyn summary file, the latter is hard to estimate before the full-system linearization of the coupled FAST model is realized. Until then, experience has shown that the highest physical mode when SubDyn is coupled to FAST is often the platform-heave mode of ElastoDyn, with a frequency given by Eq. 4, where the variables are defined in Section 4.2.

$$
\begin{gathered}
d t_{\max }=\frac{1}{10\left(f_{\max }\right)} \\
f=\frac{1}{2 \pi} \sqrt{\frac{K_{33}^{(S D)}}{\left(M_{33}^{(S D)}+M^{(E D)}\right)}}
\end{gathered}
$$

For the AB4 method, the recommend time step is half the value given by Eq. 3 .

For AM2, being implicit, the required time step is not driven by natural frequencies within SubDyn, but should still be chosen to ensure solution accuracy and that the coupling to FAST is numerically stable. 


\section{SubDyn Theory}

\subsection{Overview}

This section focuses on the theory behind the SubDyn module.

SubDyn relies on two main engineering schematizations: 1) a linear frame finite-element beam model (LFEB), and 2) a dynamics system reduction via the Craig-Bampton (C-B) method together with a static-improvement method (SIM), greatly reducing the number of modes needed to obtain an accurate solution.

Nonlinearities come from many aspects, including material nonlinearity, axial shortening caused by bending, large displacement, and so on. The material nonlinearity is not considered here because most of the offshore multimember support structures are designed to use steel and the maximum stress is intended to be below the yield strength of the material. Damiani et al. (2013) demonstrate that a linear finite-element method is suitable when analyzing wind turbine substructures. (Several wind turbine configurations that varied in base geometry, load paths, sizes, supported towers, and turbine masses were analyzed under extreme loads using nonlinear and linear models. The results revealed that the nonlinear behavior was mainly caused by the mono-tower response and had little effect on the multimember support structures.) Therefore, an LFEB model for the substructure was considered appropriate for wind turbine substructures. The LFEB can accommodate different element types, including Euler-Bernoulli and Timoshenko beam elements of either constant or longitudinally tapered cross sections (Timoshenko beam elements account for shear deformation and are better suited to represent low aspect ratio beams that may be used within frames and to transfer the loads within the frame).

The large number of DOFs $\left(\sim 10^{3}\right)$ associated with a standard finite-element analysis of a typical multimember structure would hamper computational efficiency during wind turbine system dynamic simulations. As a result, the C-B system reduction was implemented to speed up processing time while retaining a high level of fidelity in the overall system response. The C-B reduction is used to recharacterize the substructure finite-element model into a reduced DOF model that maintains the fundamental low-frequency response modes of the structure. In the SubDyn initialization step, the large substructure physical DOFs (displacements) are reduced to a small number of modal DOFs and interface (boundary) DOFs, and during each time step, only the equations of motion of these DOFs need to be solved. SubDyn only solves the equations of motion for the modal DOFs, the motion of the interface (boundary) DOFs are prescribed when running SubDyn in stand-alone mode or solved through equations of motion in ElastoDyn when SubDyn is coupled to FAST.

Retaining just a few DOFs may, however, lead to the exclusion of axial modes (normally of very high frequencies), which are important to capture static loading effects, such as those caused by gravity and buoyancy. The so-called SIM was implemented to mitigate this problem. SIM computes two static solutions at each time step: one based on the full system stiffness matrix and one based on the C-B reduced stiffness matrix. At each time step the time-varying, C-B based, dynamic solution is superimposed on the difference between the two static solutions, which amounts to quasi-statically accounting for the contribution of those modes not directly included within the dynamic solution. 
In SubDyn, the substructure is considered to be clamped at the bottom nodes (normally at the seabed) and rigidly connected to the TP at the substructure top nodes (interface nodes). Other constraints will be implemented in future releases, including a soil-pile interaction module. As described in other sections of this document, the input file defines the substructure geometry, material properties, and constraints. Users can define: element types; full finite-element mode or $\mathrm{C}-\mathrm{B}$ reduction; the number of modes to be retained in the $\mathrm{C}-\mathrm{B}$ reduction; modal damping coefficients; whether to take advantage of SIM; and the number of elements for each member.

The following sections discuss the integration of SubDyn within the FAST framework, the main coordinate systems used in the module, and the theory pertaining to the LFEB, the C-B reduction, and SIM. The state-space formulations to be used in the time-domain simulation are also presented. The last section discusses the calculation of the base reaction calculation. For further details, see also Song et al. (2013).

\subsection{Integration with the FAST Modularization Framework}

Based on a new modularization framework (Jonkman 2013) FAST joins an aerodynamics module, a hydrodynamics module, a control and electrical system (servo) module, and structuraldynamics (elastic) modules to enable coupled nonlinear aero-hydro-servo-elastic analysis of land-based and offshore wind turbines in the time domain. Figure 2 shows the basic layout of the SubDyn module within the FAST modularization framework.

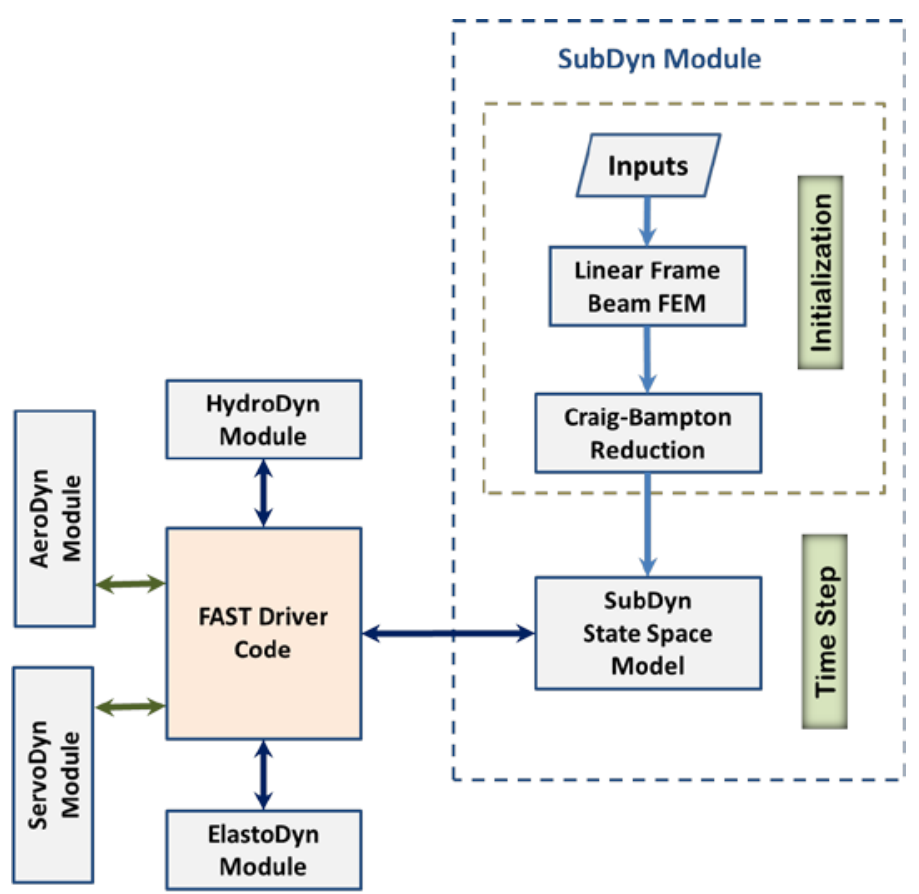

Figure 2. SubDyn layout within the modularization framework

In a loosely coupled time-integration scheme, the glue-code transfers data at each time step. Such data includes hydrodynamic loads, substructure response, loads transmitted to the TP, and TP response among SubDyn, HydroDyn, and ElastoDyn. At the interface nodes, the TP displacement, rotation, velocity, and acceleration are inputs to SubDyn from ElastoDyn, and the reaction forces at the TP are outputs of SubDyn for input to ElastoDyn. SubDyn also outputs the 
substructure displacements and velocities for input to HydroDyn to calculate the hydrodynamic loads that become inputs for SubDyn. In addition, SubDyn can calculate the member forces, as requested by the user. Within this scheme, SubDyn tracks its states and integrates its equations through its own solver.

In a tightly coupled time-integration scheme, SubDyn sets up its own equations, but its states and those of other modules are tracked and integrated by a solver within the glue-code that is common to all of the modules.

SubDyn is implemented in a state-space formulation that sets the equation of motion of the substructure system with physical DOFs at the boundaries and modal DOFs representing all interior motions. At each time step, loads and motions are exchanged between modules through the driver code; the modal responses are calculated inside SubDyn's state-space model; and the next time-step responses are calculated by the SubDyn integrator for loose coupling and the global system integrator for tight coupling.

\subsection{Coordinate Systems}

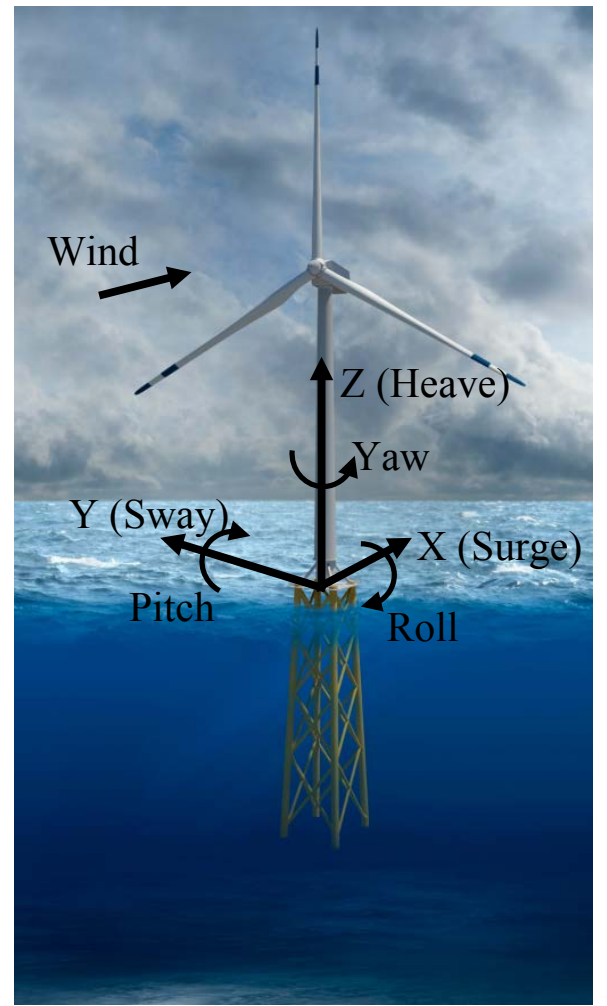

Figure 3. Global (coincident with the substructure) coordinate system. Also shown are the DOFs associated with the TP reference point. 


\subsubsection{Global and Substructure Coordinate System: $(X, Y, Z)$ or $\left(X_{S S}, Y_{S S}, Z_{S S}\right)$}

- The global axes are represented by the unit vectors $\hat{I}, \hat{J}$, and $\hat{K}$.

- The origin is set at the intersection between the undeflected tower centerline and the horizontal plane identified by the mean sea level (MSL).

- The positive $Z\left(Z_{S S}\right)$ axis is vertical and pointing upward, opposite gravity.

- The positive $X\left(X_{S S}\right)$ axis is along the nominal (zero-degree) wind and wave propagation direction.

- The $Y\left(Y_{S S}\right)$ axis is transverse and can be found assuming a right-handed Cartesian coordinate system (directed to the left when looking in the nominal downwind direction).

\subsubsection{Member or Element Local Coordinate System $\left(x_{e}, y_{e}, z_{e}\right)$}

- Axes are represented by the unit vectors $\hat{i}_{e}, \hat{j}_{e}$, and $\hat{k}_{e}$.

- The origin is set at the shear center of the cross section at the start node (S).

- The local $z_{e}$ axis is along the elastic axis of the member, directed from the start node (S) to the end node (E). Nodes are ordered along the member main axis directed from start joint to end joint (per user's input definition).

- The local $x_{e}$ axis is parallel to the global $X Y$ plane, and directed such that a positive, less than or equal to $180^{\circ}$ rotation about it, would bring the local $z_{e}$ axis parallel to the global $Z$ axis.

- The local $y_{e}$ axis can be found assuming a right-handed Cartesian coordinate system.

\subsubsection{Local to Global Transformation}

The transformation from local to global coordinate system can be expressed by the following equation:

$$
\left\{\begin{array}{l}
\Delta X \\
\Delta Y \\
\Delta Z
\end{array}\right\}=\left[\boldsymbol{D}_{C}\right]\left\{\begin{array}{l}
\Delta x_{e} \\
\Delta y_{e} \\
\Delta z_{e}
\end{array}\right\}
$$

where $\left\{\begin{array}{l}\Delta x_{e} \\ \Delta y_{e} \\ \Delta z_{e}\end{array}\right\}$ is a generic vector in the local coordinate system, and $\left\{\begin{array}{l}\Delta X \\ \Delta Y \\ \Delta Z\end{array}\right\}$ the same vector but in the global coordinate system; and $\left[\boldsymbol{D}_{C}\right]$ is the direction cosine matrix of the member axes and can be obtained as follows: 


$$
\left[\boldsymbol{D}_{C}\right]=\left[\begin{array}{ccc}
\frac{Y_{E}-Y_{S}}{L_{e x y}} & \frac{\left(X_{E}-X_{S}\right)\left(Z_{E}-Z_{S}\right)}{L_{e x y} L_{e}} & \frac{X_{E}-X_{S}}{L_{e}} \\
\frac{-X_{E}+X_{S}}{L_{e x y}} & \frac{\left(Z_{E}-Z_{S}\right)\left(Y_{E}-Y_{S}\right)}{L_{e x y} L_{e}} & \frac{Y_{E}-Y_{S}}{L_{e}} \\
0 & -\frac{L_{e x y}}{L_{e}} & \frac{Z_{E}-Z_{S}}{L_{e}}
\end{array}\right]
$$

Where $\left(X_{S}, Y_{S}, Z_{S}\right)$ and $\left(X_{E}, Y_{E}, Z_{E}\right)$ are the start and end joints of the member (or nodes of the element of interest) in global coordinate system; $L_{e x y}=\sqrt{\left(X_{E}-X_{S}\right)^{2}+\left(Y_{E}-Y_{S}\right)^{2}}$ and $L_{e}=\sqrt{\left(X_{E}-X_{S}\right)^{2}+\left(Y_{E}-Y_{S}\right)^{2}+\left(Z_{E}-Z_{S}\right)^{2}}$.

If $X_{E}=X_{S}$ and $Y_{E}=Y_{S}$, the $\left[\boldsymbol{D}_{C}\right]$ matrix can be found as follows:

if $Z_{S}<Z_{E}$ then

$$
\left[\boldsymbol{D}_{C}\right]=\left[\begin{array}{lll}
1 & 0 & 0 \\
0 & 1 & 0 \\
0 & 0 & 1
\end{array}\right]
$$

else

$$
\left[\boldsymbol{D}_{C}\right]=\left[\begin{array}{ccc}
1 & 0 & 0 \\
0 & -1 & 0 \\
0 & 0 & -1
\end{array}\right]
$$

In the current SubDyn release, the transpose (global to local) of these direction cosine matrices for each member is returned in the summary file. Given the circular shape of the member cross sections, the direction cosine matrices have little importance on the member load verification. To verify joints following the standards (e.g., ISO 19902, API RP-2A); however, the bending moments need to be decomposed into in-plane and out-of-plane components, where the plane is that defined by either a pair of braces (for an X-joint), or by the pair brace(s) plus leg (for a Kjoint). It is therefore important to have the direction cosines of the interested members readily available to properly manipulate and transform the local shear forces and bending moments.

In future releases, where member cross sections other than circular will be allowed, the user will need to input cosine matrices to indicate the final orientation of the member principal axes with respect to the global reference frame. 


\subsection{Linear Finite-Element Beam Model}

In SubDyn, the LFEB can accommodate different two-node beam element types, including Euler-Bernoulli and Timoshenko beam elements, either of constant or tapered cross sections. The tapered element formulation has been derived, but has not been implemented in the current SubDyn release.

The uniform and tapered Euler-Bernoulli beam elements are displacement-based and use thirdorder interpolation functions that guarantee the displacement and rotation continuity between elements. The uniform Timoshenko beam element is derived by introducing the shear deformation into the uniform Euler-Bernoulli element, so the displacements are represented by third-order interpolation functions as well.

\subsubsection{Element Formulation}

Following the classic Timoshenko beam theory, the generic two-node element stiffness and consistent mass matrices can be written as follows (see, for instance, Panzer et al. 2009):

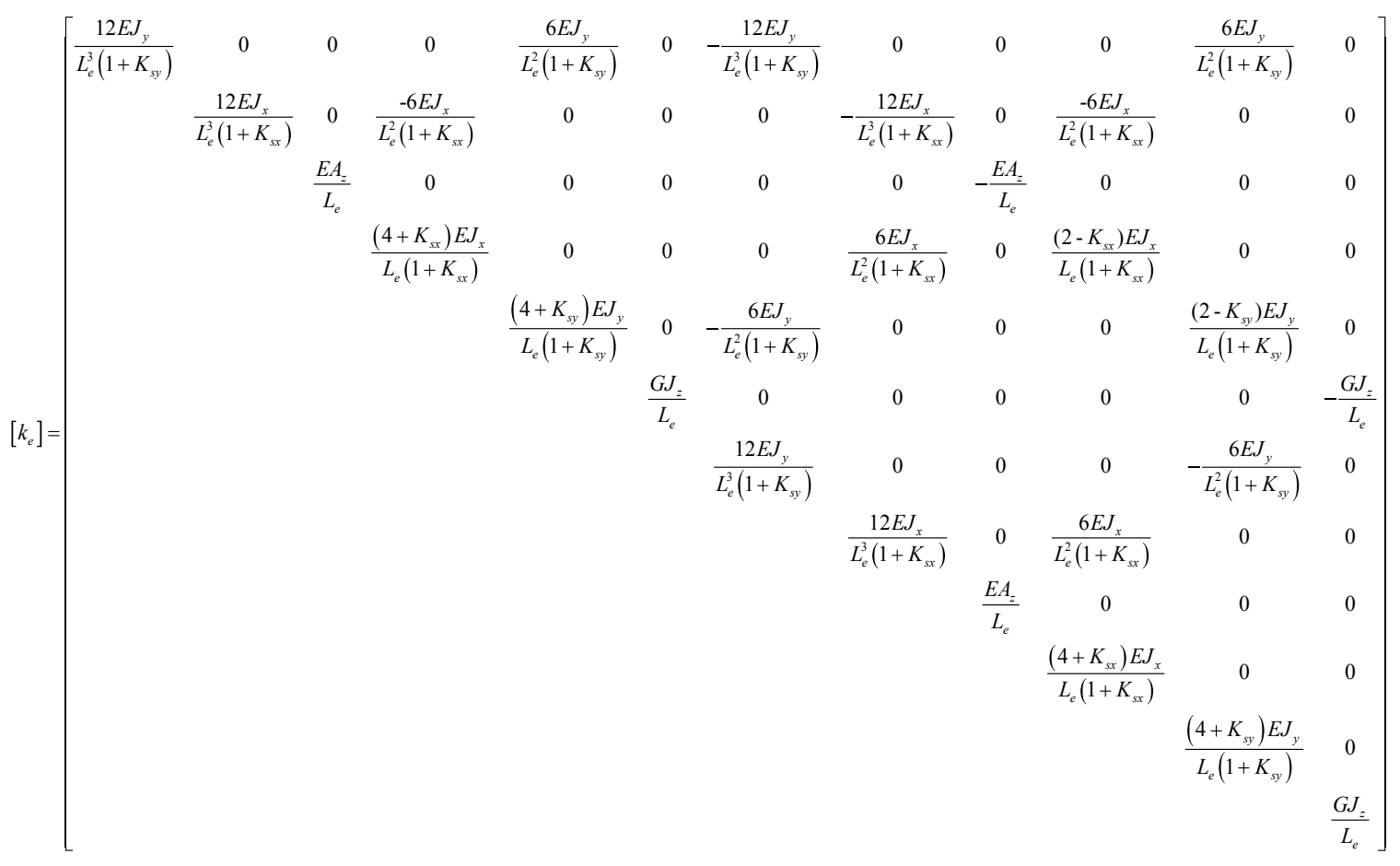




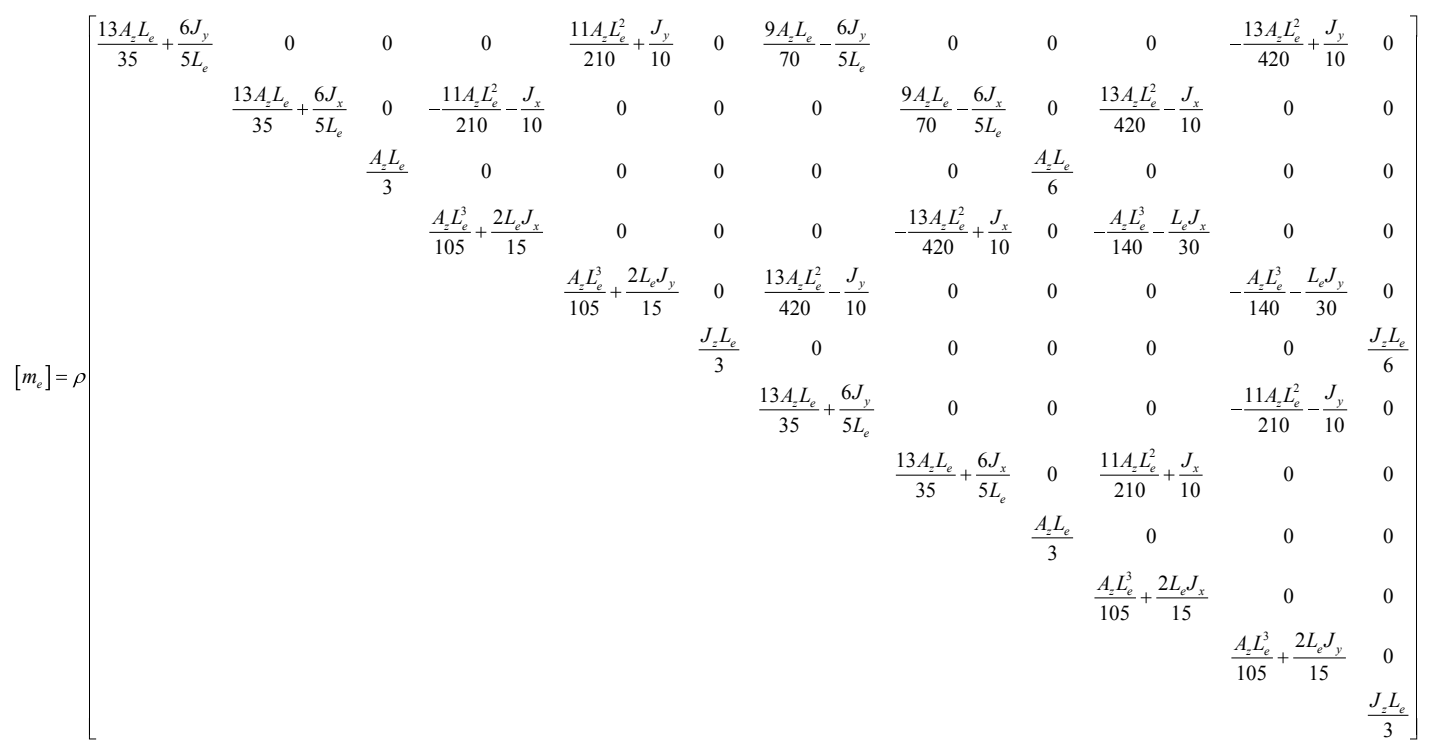

where the matrices are symmetric, and where $A_{z}$ is the element cross-section area, $J_{x}, J_{y}, J_{z}$ are the area second moments of inertia with respect to principal axes of the cross section; $L_{e}$ is the length of the undisplaced element from start-node to end-node; $\rho, E$, and $G$ are material density, Young's, and Shear moduli, respectively; $K_{s x}, K_{s y}$ are shear correction factors as shown below (they are set to zero if the E-B formulation is chosen):

$$
K_{s x}=\frac{12 E J_{y}}{G A_{s x} L_{e}^{2}}, K_{s y}=\frac{12 E J_{x}}{G A_{s y} L_{e}^{2}}
$$

where the shear areas along the local $x$ and $y$ (principal) axes are defined as:

$$
\begin{aligned}
& A_{s x}=k_{a x} A_{z} \\
& A_{s y}=k_{a y} A_{z}
\end{aligned}
$$

and

$$
k_{a x}=k_{a y}=\frac{6(1+v)^{2}\left(1+\left(\frac{D_{i}}{D_{o}}\right)^{2}\right)^{2}}{\left(1+\left(\frac{D_{i}}{D_{o}}\right)^{2}\right)^{2}\left(7+14 v+8 v^{2}\right)+4\left(\frac{D_{i}}{D_{o}}\right)^{2}\left(5+10 v+4 v^{2}\right)}
$$

Eq. 13 is from Steinboeck et al. (2013) for hollow circular cross sections, with $v$ denoting Poisson's ratio.

Before assembling the global system stiffness $(K)$ and mass $(M)$ matrices, the individual $\left[k_{e}\right]$ and $\left[m_{e}\right]$ are modified via $\left[\boldsymbol{D}_{C}\right]$ as shown in the following equations: 


$$
\begin{gathered}
{[k]=\left[\begin{array}{cccc}
{\left[\boldsymbol{D}_{C}\right]} & 0 & 0 & 0 \\
& {\left[\boldsymbol{D}_{C}\right]} & 0 & 0 \\
& & {\left[\boldsymbol{D}_{C}\right]} & 0 \\
& & & {\left[\boldsymbol{D}_{C}\right]}
\end{array}\right]\left[k_{e}\right]\left[\begin{array}{cccc}
{\left[\boldsymbol{D}_{C}\right]} & 0 & 0 & 0 \\
& {\left[\boldsymbol{D}_{C}\right]} & 0 & 0 \\
& {\left[\boldsymbol{D}_{C}\right]} & 0 \\
& & & {\left[\boldsymbol{D}_{C}\right]}
\end{array}\right]} \\
{[m]=\left[\begin{array}{cccc}
{\left[\boldsymbol{D}_{C}\right]} & 0 & 0 & 0 \\
& {\left[\boldsymbol{D}_{C}\right]} & 0 & 0 \\
& & {\left[\boldsymbol{D}_{C}\right]} & 0 \\
& & & {\left[\boldsymbol{D}_{C}\right]}
\end{array}\right]\left[m_{e}\right]\left[\begin{array}{cccc}
{\left[\boldsymbol{D}_{C}\right]} & 0 & 0 & 0 \\
& {\left[\boldsymbol{D}_{C}\right]} & 0 & 0 \\
& & {\left[\boldsymbol{D}_{C}\right]} & 0 \\
& & & {\left[\boldsymbol{D}_{C}\right]}
\end{array}\right]}
\end{gathered}
$$

where $m$ and $k$ are element matrices in the global coordinate system.

\subsubsection{Self-Weight Loads}

The loads caused by self-weight are pre-computed during initialization. It is therefore assumed that the displacements will be small and that P- $\Delta$ effects are small for the substructure. For a nontapered beam element, the lumped loads caused by gravity to be applied at the end nodes are as follows (in the global coordinate system):

$$
\left\{F_{G}\right\}=\rho A_{z} g\left\{\begin{array}{c}
0 \\
0 \\
-\frac{L_{e}}{2} \\
-\frac{L_{e}^{2}}{12} D_{c 2,3} \\
\frac{L_{e}^{2}}{12} D_{c 1,3} \\
0 \\
0 \\
0 \\
-\frac{L_{e}}{2} \\
\frac{L_{e}^{2}}{12} D_{c 2,3} \\
-\frac{L_{e}^{2}}{12} D_{c 1,3} \\
0
\end{array}\right\}
$$

Note also that if lumped masses exist (selected by the user at prescribed joints), their contribution will be included as concentrated forces along global $Z$ at the relevant nodes. 


\subsection{Dynamic System of Equations and C-B Reduction}

The main equations of motion of SubDyn are written as follows:

$$
[M]\{\ddot{U}\}+[C]\{\dot{U}\}+[K]\{U\}=\{F\}
$$

where $[M]$ and $[K]$ are the global mass and stiffness matrices of the substructure beam frame, assembled from the element mass and stiffness matrices. $\{U\}$ and $\{F\}$ are the displacements and external forces along all of the DOFs of the assembled system. The damping matrix $[C]$ is not assembled from the element contributions, because those are normally unknown, but treated from a system point of view as shown in the following paragraphs. A derivative with respect to time is represented by a dot, so that $\dot{U}$ and $\ddot{U}$ are the first- and second-time derivatives of $U$, respectively.

The number of DOFs associated with Eq. 17 can easily grow to the thousands for typical beam frame substructures. That factor, combined with the need for time-domain simulations of turbine dynamics, may seriously slow down the computational efficiency of aeroelastic codes such as FAST (note that a typical wind turbine system model in ElastoDyn has about 20 DOFs). For this reason, a C-B methodology was used to recharacterize the substructure finite-element model into a reduced DOF model that maintains the fundamental low-frequency response modes of the structure. With the C-B method, the DOFs of the substructure can be reduced to about 10 (user defined, see also Section 4.4). This system reduction method was first introduced by Hurty (1964) and later expanded by Craig and Bampton (1968).

In SubDyn's C-B reduction, the substructure nodes are separated into two groups: 1) the boundary nodes (identified with a subscript " $R$ " in what follows) that include the nodes restrained at the base of the structure and the interface nodes; and 2) the interior nodes (or leftover nodes, identified with a subscript " $L$ "). The interface nodes are assumed rigidly connected among one another and to the TP reference point.

The derivation of the system reduction is shown below. After the LFEB assembly, the system equation of motion of Eq. 17 can be partitioned as follows:

$$
\left[\begin{array}{ll}
M_{R R} & M_{R L} \\
M_{L R} & M_{L L}
\end{array}\right]\left\{\begin{array}{l}
\ddot{U}_{R} \\
\ddot{U}_{L}
\end{array}\right\}+\left[\begin{array}{ll}
C_{R R} & C_{R L} \\
C_{L R} & C_{L L}
\end{array}\right]\left\{\begin{array}{l}
\dot{U}_{R} \\
\dot{U}_{L}
\end{array}\right\}+\left[\begin{array}{cc}
K_{R R} & K_{R L} \\
K_{L R} & K_{L L}
\end{array}\right]\left\{\begin{array}{l}
U_{R} \\
U_{L}
\end{array}\right\}=\left\{\begin{array}{l}
F_{R}+F_{R g} \\
F_{L}+F_{L g}
\end{array}\right\}
$$

where the subscript $R$ denotes the boundary DOFs (there are R DOFs), and the subscript $L$ the interior DOFs (there are $L$ DOFs).

In Eq. 18, the applied forces include external forces (e.g., hydrodynamic forces and those transmitted through the TP to the substructure) $\left(F_{R}, F_{L}\right)$ and gravity forces $\left(F_{R g}, F_{L g}\right)$, which are considered static forces lumped at each node. The forces at the boundary nodes can be broken down into hydrodynamic forces and those transferred to and from ElastoDyn via the TP, thus: 


$$
F_{R}=F_{H D R}+F_{E D R}
$$

The fundamental assumption of the C-B method is that the contribution to the displacement of the interior nodes can be simply approximated by a subset $q_{m}(m \leq L)$ of the interior generalized DOFs $\left(q_{L}\right)$. The relationship between physical DOFs and generalized DOFs can be written as:

$$
\left\{\begin{array}{l}
U_{R} \\
U_{L}
\end{array}\right\}=\left[\begin{array}{cc}
I & 0 \\
\Phi_{R} & \Phi_{L}
\end{array}\right]\left\{\begin{array}{c}
U_{R} \\
q_{L}
\end{array}\right\}
$$

$\Phi_{R}(L \times R$ matrix $)$ represents the physical displacements of the interior nodes for static, rigid body motions at the boundary (interface nodes' DOFs, because the restrained nodes' DOFs are locked by definition). By considering the homogeneous, static version of Eq. 18, the second row can be manipulated to yield:

$$
\left[K_{L R}\right]\left\{U_{R}\right\}+\left[K_{L L}\right]\left\{U_{L}\right\}=\{0\}
$$

Rearranging Eq. 21, and making use of Eq. 20 and assuming rigid motion $\left(q \_l=\{0\}\right)$, yields:

$$
\Phi_{R}=-K_{L L}^{-1} K_{L R}
$$

where the brackets have been removed for simplicity.

$\Phi_{L}(L \times L$ matrix $)$ represents the internal eigenmodes, i.e., the natural modes of the system restrained at the boundary (interface and bottom nodes), and can be obtained by solving the eigenvalue problem:

$$
K_{L L} \Phi_{L}=\omega^{2} M_{L L} \Phi_{L}
$$

The eigenvalue problem in Eq. 23 leads to the reduced basis of generalized modal DOFs $q_{m}$, which are chosen as the first few $(m)$ eigenvectors that are arranged by increasing eigenfrequencies. $\Phi_{L}$ is mass normalized, so that:

$$
\Phi_{L}^{T} M_{L L} \Phi_{L}=I
$$

where $I$ is the identity matrix.

By then reducing the number of generalized DOFs to $m(\leq L), \Phi_{m}$ is chosen to denote the truncated set of $\Phi_{L}$ (keeping $m$ of the total internal modes, hence $m$ columns), and $\Omega_{m}$ is the diagonal $(m \times m)$ matrix containing the corresponding eigenfrequencies. In SubDyn, the user decides how many modes to retain, including possibly zero or all modes. Retaining zero modes corresponds to a Guyan (static) reduction; retaining all modes corresponds to keeping the full finite-element model.

The C-B transformation is therefore represented by:

$$
\left\{\begin{array}{l}
U_{R} \\
U_{L}
\end{array}\right\}=\left[\begin{array}{cc}
I & 0 \\
\Phi_{R} & \Phi_{m}
\end{array}\right]\left\{\begin{array}{l}
U_{R} \\
q_{m}
\end{array}\right\}
$$


By using Eq. 25, the interior DOFs are hence transformed from physical DOFs to modal DOFs, and by pre-multiplying both sides of Eq. 18 by $\left[\begin{array}{cc}I & 0 \\ \Phi_{R} & \Phi_{m}\end{array}\right]^{T}=\left[\begin{array}{cc}I & \Phi_{R}^{T} \\ 0 & \Phi_{m}^{T}\end{array}\right]$, and making use of Eq. 24, Eq. 18 can be rewritten as:

$$
\left[\begin{array}{cc}
M_{B B} & M_{B m} \\
M_{m B} & I
\end{array}\right]\left\{\begin{array}{l}
\ddot{U}_{R} \\
\ddot{q}_{m}
\end{array}\right\}+\left[\begin{array}{cc}
0 & 0 \\
0 & 2 \zeta \Omega_{m}
\end{array}\right]\left\{\begin{array}{c}
\dot{U}_{R} \\
\dot{q}_{m}
\end{array}\right\}+\left[\begin{array}{cc}
K_{B B} & 0 \\
0 & \Omega_{m}^{2}
\end{array}\right]\left\{\begin{array}{l}
U_{R} \\
q_{m}
\end{array}\right\}=\left\{\begin{array}{c}
\left(F_{R}+F_{R g}\right)+\Phi_{R}^{T}\left(F_{L}+F_{L g}\right) \\
\Phi_{m}^{T}\left(F_{L}+F_{L g}\right)
\end{array}\right\}
$$

Eq. 26 assumes that:

$\left[\begin{array}{cc}I & \Phi_{R}^{T} \\ 0 & \Phi_{m}^{T}\end{array}\right]\left[\begin{array}{cc}C_{R R} & C_{R L} \\ C_{L R} & C_{L L}\end{array}\right]\left[\begin{array}{cc}I & 0 \\ \Phi_{R} & \Phi_{m}\end{array}\right]=\left[\begin{array}{cc}C_{R R}+C_{R L} \Phi_{R}+\Phi_{R}^{T} C_{L R}+\Phi_{R}^{T} C_{L L} \Phi_{R} & C_{R L} \Phi_{m}+\Phi_{R}^{T} C_{L L} \Phi_{m} \\ \Phi_{m}^{T} C_{L R}+\Phi_{m}^{T} C_{L L} \Phi_{R} & \Phi_{m}^{T} C_{L L} \Phi_{m}\end{array}\right]=\left[\begin{array}{cc}0 & 0 \\ 0 & \Phi_{m}^{T} C_{L L} \Phi_{m}\end{array}\right]=\left[\begin{array}{cc}0 & 0 \\ 0 & 2 \zeta \Omega_{m}\end{array}\right]$

In other words, the only damping matrix term retained is the one associated with internal DOF damping. This assumption has implications on the damping at the interface with the turbine system, as discussed in Section 6.2. The diagonal $(m \times m)$ matrix $\zeta$ contains the modal damping ratios corresponding to each retained internal mode. In SubDyn, the user provides damping ratios (in percent of critical damping coefficients) for the retained modes.

The matrix partitions in Eq. 26 are calculated as follows:

$$
\begin{aligned}
& M_{B B}=M_{R R}+M_{R L} \Phi_{R}+\Phi_{R}^{T} M_{L R}+\Phi_{R}^{T} M_{L L} \Phi_{R} \\
& M_{m B}=\Phi_{m}^{T} M_{L R}+\Phi_{m}^{T} M_{L L} \Phi_{R} \\
& M_{B m}=M_{m B}^{T} \\
& K_{B B}=K_{R R}+K_{R L} \Phi_{R}
\end{aligned}
$$

Next, the boundary nodes are partitioned into those at the interface, $\bar{U}_{R}$, and those at the bottom, which are fixed:

$$
U_{R}=\left[\begin{array}{c}
\bar{U}_{R} \\
0
\end{array}\right]
$$

The overhead bar here and below denotes matrices/vectors after the fixed-bottom boundary conditions are applied. The interface nodes are treated as rigidly connected to the TP, hence it is convenient to use rigid-body TP DOFs (one node with 6 DOFs at the TP reference point) in place of the interface DOFs. The interface DOFs, $\bar{U}_{R}$, and the TP DOFs are related to each other as follows:

$$
\bar{U}_{R}=T_{I} U_{T P}
$$

where $T_{I}$ is a $(6 \cdot N I N) \times 6$ matrix, NIN is the number of interface nodes, and $U_{T P}$ is the 6 DOFs of the rigid transition piece. The matrix $T_{l}$ can be written as follows: 


$$
T_{I}=\left[\begin{array}{cccccc}
1 & 0 & 0 & 0 & \Delta Z_{1} & -\Delta Y_{1} \\
0 & 1 & 0 & -\Delta Z_{1} & 0 & \Delta X_{1} \\
0 & 0 & 1 & \Delta Y_{1} & -\Delta X_{1} & 0 \\
0 & 0 & 0 & 1 & 0 & 0 \\
0 & 0 & 0 & 0 & 1 & 0 \\
0 & 0 & 0 & 0 & 0 & 1 \\
\vdots & \vdots & \vdots & \vdots & \vdots & \vdots \\
1 & 0 & 0 & 0 & \Delta Z_{i} & -\Delta Y_{i} \\
0 & 1 & 0 & -\Delta Z_{i} & 0 & \Delta X_{i} \\
0 & 0 & 1 & \Delta Y_{i} & -\Delta X_{i} & 0 \\
0 & 0 & 0 & 1 & 0 & 0 \\
0 & 0 & 0 & 0 & 1 & 0 \\
0 & 0 & 0 & 0 & 0 & 1 \\
\vdots & \vdots & \vdots & \vdots & \vdots & \vdots
\end{array}\right], \quad(i=1,2, \cdots, N I N)
$$

with

$$
\begin{aligned}
\Delta X_{i} & =X_{I N i}-X_{T P} \\
\Delta Y_{i} & =Y_{I N i}-Y_{T P} \\
\Delta Z_{i} & =Z_{I N i}-Z_{T P}
\end{aligned}
$$

where $\left(X_{I N i}, Y_{I N}, Z_{I N}\right)$ are the coordinates of $i^{i l}$ interface node and $\left(X_{T P}, Y_{T P}, Z_{T P}\right)$ are the coordinates of the TP reference point within the global coordinate system.

In terms of TP DOFs, the system equation of motion Eq. 26 after the boundary constraints are applied (the rows and columns corresponding to the DOFs of the nodes that are restrained at the seabed are removed from the equation of motion) becomes:

$$
\left[\begin{array}{cc}
\tilde{M}_{B B} & \tilde{M}_{B m} \\
\tilde{M}_{m B} & I
\end{array}\right]\left\{\begin{array}{c}
\ddot{U}_{T P} \\
\ddot{q}_{m}
\end{array}\right\}+\left[\begin{array}{cc}
0 & 0 \\
0 & 2 \zeta \Omega_{m}
\end{array}\right]\left\{\begin{array}{c}
\dot{U}_{T P} \\
\dot{q}_{m}
\end{array}\right\}+\left[\begin{array}{cc}
\tilde{K}_{B B} & 0 \\
0 & \Omega_{m}^{2}
\end{array}\right]\left\{\begin{array}{c}
U_{T P} \\
q_{m}
\end{array}\right\}=\left\{\begin{array}{c}
\tilde{F}_{T P} \\
\tilde{F}_{m}
\end{array}\right\}
$$

with

$$
\begin{aligned}
& \tilde{M}_{B B}=T_{I}^{T} \bar{M}_{B B} T_{I} \\
& \tilde{M}_{B m}=T_{I}^{T} \bar{M}_{B m} \\
& \tilde{M}_{m B}=\tilde{M}_{B m}^{T} \\
& \tilde{K}_{B B}=T_{I}^{T} \bar{K}_{B B} T_{I} \\
& \tilde{F}_{T P}=F_{T P}+T_{I}^{T} \bar{F}_{H D R}+T_{I}^{T} \bar{F}_{R g}+T_{I}^{T} \bar{\Phi}_{R}^{T}\left(F_{L}+F_{L g}\right) \\
& \tilde{F}_{m}=\Phi_{m}^{T}\left(F_{L}+F_{L g}\right)
\end{aligned}
$$

where the TP reaction force, i.e., the force applied to the substructure through the TP, is denoted by:

$$
F_{T P}=T_{I}^{T} F_{E D R}
$$

Equation 32 represents the equations of motion of the substructure after the $\mathrm{C}-\mathrm{B}$ reduction. The total DOFs of the substructure are reduced from ( $6 \times$ total number of nodes) to $(6+m)$. 
During initialization, SubDyn calculates: the parameter matrices $\tilde{M}_{B B}, \tilde{M}_{m B}, \tilde{M}_{B m}, \tilde{K}_{B B}, \Phi_{m}, \Phi_{R}, T_{I}$; the gravity arrays $\bar{F}_{R g}$ and $F_{L g}$; and the internal frequency matrix $\Omega_{m}$. The substructure response at each time step can then be obtained by using the state-space formulation discussed in the next section.

\subsubsection{State-Space Formulation}

A state-space formulation of the substructure structural dynamics problem was devised to integrate SubDyn within the FAST modularization framework. The state-space formulation was developed in terms of inputs, outputs, states, and parameters. The notations highlighted here are consistent with those used in Jonkman (2013). Inputs (identified by $u$ ) are a set of values supplied to SubDyn that, along with the states, are needed to calculate future states and the system's output. Outputs $(y)$ are a set of values calculated by and returned from SubDyn that depend on the states, inputs, and/or parameters through output equations (with functions $Y$ ). States are a set of internal values of SubDyn that are influenced by the inputs and used to calculate future state values and the output. In SubDyn, only continuous states are considered. Continuous states $(x)$ are states that are differentiable in time and characterized by continuous time differential equations (with functions $X$ ). Parameters $(p)$ are a set of internal system values that are independent of the states and inputs. Furthermore, parameters can be fully defined at initialization and characterize the system's state equations and output equations.

In SubDyn, the inputs are defined as:

$$
u=\left\{\begin{array}{l}
u_{1} \\
u_{2} \\
u_{3} \\
u_{4} \\
u_{5}
\end{array}\right\}=\left\{\begin{array}{l}
U_{T P} \\
\dot{U}_{T P} \\
\ddot{U}_{T P} \\
F_{L} \\
F_{H D R}
\end{array}\right\}
$$

where $F_{L}$ are the hydrodynamic forces on every interior node of the substructure from HydroDyn, and $F_{H D R}$ are the analogous forces at the boundary nodes; $U_{T P}, \dot{U}_{T P}$, and $\ddot{U}_{T P}$ are TP deflections (6 DOFs), velocities, and accelerations, respectively. For SubDyn in stand-alone mode (uncoupled from FAST), $F_{L}$ is assumed to be zero.

In first-order form, the states are defined as:

$$
x=\left\{\begin{array}{l}
x_{1} \\
x_{2}
\end{array}\right\}=\left\{\begin{array}{l}
q_{m} \\
\dot{q}_{m}
\end{array}\right\}
$$

From the system equation of motion, the state equation corresponding to Eq. 32 can be written as a standard linear system state equation:

$$
\dot{x}=X=A x+B u+F_{X}
$$

where

$$
A=\left[\begin{array}{cc}
0 & I \\
-\Omega_{m}^{2} & -2 \zeta \Omega_{m}
\end{array}\right], B=\left[\begin{array}{ccccc}
0 & 0 & 0 & 0 & 0 \\
0 & 0 & -\tilde{M}_{m B} & \Phi_{m}^{T} & 0
\end{array}\right], F_{X}=\left\{\begin{array}{c}
0 \\
\Phi_{m}^{T} F_{L g}
\end{array}\right\}
$$


In SubDyn, the outputs to the ElastoDyn module are the reaction forces at the transition piece $F_{T P}$ :

$$
y_{1}=Y_{1}=-F_{T P}
$$

By examining Eq. 32, the output equation for $y_{1}$ can be found as:

$$
-Y_{1}=C_{1} x+D_{1} \bar{u}+F_{Y 1}
$$

where

$$
\begin{aligned}
& C_{1}=\left[\begin{array}{ll}
-\tilde{M}_{B m} \Omega_{m}^{2} & -2 \tilde{M}_{B m} \zeta \Omega_{m}
\end{array}\right] \\
& D_{1}=\left[\begin{array}{lllll}
\tilde{K}_{B B} & 0 & \tilde{M}_{B B}-\tilde{M}_{B m} \tilde{M}_{m B} & \tilde{M}_{B m} \Phi_{m}^{T}-T_{I}^{T} \bar{\Phi}_{R}^{T} & -T_{I}^{T}
\end{array}\right] \\
& F_{Y 1}=\tilde{M}_{B m} \Phi_{m}^{T} F_{L g}-T_{I}^{T}\left(\bar{F}_{R g}+\bar{\Phi}_{R}^{T} F_{L g}\right) \\
& \bar{u}=\left\{\begin{array}{l}
U_{T P} \\
\dot{U}_{T P} \\
\ddot{U}_{T P} \\
F_{L} \\
\bar{F}_{H D R}
\end{array}\right\}
\end{aligned}
$$

Note that the overbar is used on the input vector to denote that the forces apply to the interface nodes only.

The outputs to HydroDyn and other modules are the deflections, velocities, and accelerations of the substructure:

$$
y_{2}=Y_{2}=\left\{\begin{array}{c}
\bar{U}_{R} \\
U_{L} \\
\dot{\bar{U}}_{R} \\
\dot{U}_{L} \\
\ddot{\bar{U}}_{R} \\
\ddot{U}_{L}
\end{array}\right\}
$$

The output equation for $y_{2}$ can then be written as:

$$
Y_{2}=C_{2} x+D_{2} u+F_{Y 2}
$$

where 


$$
\begin{aligned}
C_{2} & =\left[\begin{array}{ll}
0 & 0 \\
\Phi_{m} & 0 \\
0 & 0 \\
0 & \Phi_{m} \\
0 & 0 \\
-\Phi_{m} \Omega_{m}^{2} & -2 \Phi_{m} \zeta \Omega_{m}
\end{array}\right], D_{2}=\left[\begin{array}{ccccc}
T_{I} & 0 & 0 & 0 & 0 \\
\bar{\Phi}_{R} T_{I} & 0 & 0 & 0 & 0 \\
0 & T_{I} & 0 & 0 & 0 \\
0 & \bar{\Phi}_{R} T_{I} & 0 & 0 & 0 \\
0 & 0 & T_{I} & 0 & 0 \\
0 & 0 & \bar{\Phi}_{R} T_{I}-\Phi_{m} \tilde{M}_{m B} & \Phi_{m} \Phi_{m}^{T} 0
\end{array}\right], \\
F_{Y 2} & =\left[\begin{array}{c}
0 \\
0 \\
0 \\
0 \\
0 \\
\Phi_{m} \Phi_{m}^{T} F_{L g}
\end{array}\right]
\end{aligned}
$$

\subsubsection{Member Force Calculation}

SubDyn can also calculate member forces by starting from the forces computed at the nodes of the elements that are contained in the member as:

Element Inertia load: $F_{I}^{e}=[m] \ddot{U}^{e}$

Element Static load: $F_{S}^{e}=[k] U^{e}$

where $[k]$ and $[m]$ are element stiffness and mass matrices, respectively. And $U_{e}$ and $\ddot{U}_{e}$ are element nodal deflections and accelerations respectively, which can be obtained from Eq. 42.

There is no good way to quantify the damping forces for each element, so the element damping forces are not calculated.

\subsubsection{Reaction Calculation}

The reactions at the base of the structure are the member forces at the base nodes. These are usually provided in member local reference frames. Additionally, the user may request an overall reaction $\vec{R}$ (six forces and moments) lumped at the center of the substructure (tower centerline) and mudline, i.e., at the reference point $(0,0,-$ WtrDpth $)$ in the global reference frame, with WtrDpth denoting the water depth. $\vec{R}$ is a six-element array that can be calculated in matrix form as follows:

$$
\vec{R}=\left[\begin{array}{c}
F_{X} \\
\vdots \\
M_{Z}
\end{array}\right]=T_{\text {React }} F_{\text {React }}
$$

where $F_{\text {React }}$ is a $\left(6 N_{\text {react }}\right)$ array containing the forces and moments at the $N_{\text {react }}$ restrained nodes in the global coordinate frame, and $T_{\text {React }}$ is a $\left(6 \times 6 N_{\text {react }}\right)$ matrix, as follows: 


$$
T_{\text {React }}=\left[\begin{array}{ccccccccccccc}
1 & 0 & 0 & 0 & 0 & 0 & \cdots & 1 & 0 & 0 & 0 & 0 & 0 \\
0 & 1 & 0 & 0 & 0 & 0 & \cdots & 0 & 1 & 0 & 0 & 0 & 0 \\
0 & 0 & 1 & 0 & 0 & 0 & \cdots & 0 & 0 & 1 & 0 & 0 & 0 \\
0 & -\Delta Z_{1} & \Delta Y_{1} & 1 & 0 & 0 & \cdots & 0 & -\Delta Z_{\text {Nreact }} & \Delta Y_{\text {Nreact }} & 1 & 0 & 0 \\
\Delta Z_{1} & 0 & -\Delta X_{1} & 0 & 1 & 0 & \cdots & \Delta Z_{\text {Nreact }} & 0 & -\Delta X_{\text {Nreact }} & 0 & 1 & 0 \\
-\Delta Y_{1} & \Delta X_{1} & 0 & 0 & 0 & 1 & \cdots & -\Delta Y_{\text {Nreact }} & \Delta X_{\text {Nreact }} & 0 & 0 & 0 & 1
\end{array}\right]
$$

where $\Delta X_{i}, \Delta Y_{i}$, and $\Delta Z_{i}\left(i=1 . . N_{\text {react }}\right)$ are coordinates of the boundary nodes with respect to the reference point. For each element with a restrained node, $F_{\text {React }}$ is calculated starting from $F_{S}^{e}$ (see Eq. 45) subtracting out the contributions of gravity $\left(F_{G}\right.$, see Eq. 16$)$ and hydrodynamic loads $\left(F_{H D R}\right)$ at the restrained node. No direct element-level inertial or damping effect is therefore included in the reaction calculation.

\subsubsection{Time Integration}

At time $t=0$, the initial states $x(0)$ are specified as initial conditions (all assumed to be zero in SubDyn) and the initial inputs are supplied to SubDyn. During each subsequent time step, the inputs and states are known values, with the inputs $u(t)$ coming from ElastoDyn and HydroDyn, and the states $x(t)$ known from the previous time-step integration. All of the parameter matrices are calculated in the SubDyn initiation module. With known $u(t)$ and $x(t), \dot{x}(t)$ can be calculated using the state equation $\dot{x}(t)=X(u, x, t)$ (see Eq. 37), and the outputs $y_{1}(t)$ and $y_{2}(t)$ can be calculated solving Eqs. 40 and 43. The element forces can also be calculated using Eq. 45. The next time-step states $x(t+\Delta t)$ are obtained by integration:

$$
[u(t), \dot{x}(t), x(t)] \stackrel{\text { Integrate }}{\longrightarrow} x(t+\Delta t)
$$

For loose coupling, SubDyn uses its own integrator, whereas for tight coupling, the states from all the modules will be integrated simultaneously using an integrator in the glue-code. SubDyn's built-in time integrator options for loose coupling are:

- Fourth-order explicit Runge-Kutta

- Fourth-order explicit Adams-Bashforth predictor

- Fourth-order explicit Adams-Bashforth-Moulton predictor-corrector

- Implicit second-order Adams-Moulton.

For more information, consult any numerical methods reference, e.g., Chapra and Canale (2010).

\subsubsection{Static-Improvement Method}

To account for the effects of static gravity (member self-weight) and buoyancy forces, one would have to include all of the structural axial modes in the C-B reduction. This inclusion often translates into hundreds of modes to be retained for practical problems. An alternative method is 
thus promoted to reduce this limitation and speed up SubDyn. This method is denoted as SIM, and computes two static solutions at each time step: one based on the full system stiffness matrix and one based on the reduced stiffness matrix. The dynamic solution then proceeds as described in the previous sections, and at each time step the time-varying dynamic solution is superimposed on the difference between the two static solutions, which amounts to quasistatically accounting for the contribution of those modes not directly included within the dynamic solution.

Recalling the previous C-B formulation Eq. 25, and adding the total static deflection of all the internal DOFs $\left(U_{L 0}\right)$, and subtracting the static deflection associated with C-B modes $\left(U_{L 0 m}\right)$, the SIM formulation is cast as in Eq. 49:

$$
U_{L}=\hat{U}_{L}+U_{L 0}-U_{L 0 m}=\underbrace{\Phi_{R} U_{R}+\Phi_{m} q_{m}}_{\hat{U}_{L}}+U_{L 0}-U_{L 0 m}
$$

Eq. 49 can be rewritten as:

$$
\left\{\begin{array}{l}
U_{R} \\
U_{L}
\end{array}\right\}=\left[\begin{array}{cccc}
I & 0 & 0 & 0 \\
\Phi_{R} & \Phi_{m} & \Phi_{L} & -\Phi_{m}
\end{array}\right]\left\{\begin{array}{c}
U_{R} \\
q_{m} \\
q_{L 0} \\
q_{m 0}
\end{array}\right\}
$$

with:

$$
\begin{gathered}
U_{L 0}=\Phi_{L} q_{L 0} \\
U_{L 0 m}=\Phi_{m} q_{m 0}
\end{gathered}
$$

where $q_{m 0}$ and $q_{L 0}$ are the $m$ and $L$ modal coefficients that are assumed to be operating in a static fashion. For Eq. 50-52 to be valid, $U_{L 0}$ and $U_{L 0 m}$ are calculated under the C-B hypothesis that the boundary nodes are fixed.

The static displacement vectors can also be calculated as follows:

$$
K_{L L} U_{L 0}=F_{L}+F_{L g}
$$

By making use of Eq. 51, and by pre-multiplying both sides times $\Phi_{L}^{T}$, Eq. 53 can be rewritten as: $\Phi_{L}^{T} K_{L L} \Phi_{L} q_{L 0}=\Phi_{L}^{T}\left\{F_{L}+F_{L g}\right\}=\tilde{F}_{L}$ or, recalling that $\Phi_{L}^{T} K_{L L} \Phi_{L}=\Omega_{L}^{2}$, as: $\Omega_{L}^{2} q_{L 0}=\tilde{F}_{L}$, or equivalently in terms of $U_{L 0}$ :

$$
U_{L 0}=\Phi_{L}\left[\Omega_{L}^{2}\right]^{-1} \tilde{F}_{L}
$$

Similarly:

$$
U_{L 0 m}=\Phi_{m}\left[\Omega_{m}^{2}\right]^{-1} \tilde{F}_{m}
$$

Note that: $\dot{U}_{L 0}=\dot{q}_{L 0}=\dot{U}_{L 0 m}=\dot{q}_{m 0}=0$ and $\ddot{U}_{L 0}=\ddot{q}_{L 0}=\ddot{U}_{L 0 m}=\ddot{q}_{m 0}=0$. 
The dynamic component $\hat{U}=\left\{\begin{array}{l}\hat{U}_{R} \\ \hat{U}_{L}\end{array}\right\}$ is calculated following the usual procedure described in Sections 5.5.1-5.5.4. For example, states are still calculated and integrated as in Eq. 37, and the output to ElastoDyn, i.e., the reaction provided by the substructure at the TP interface, is also calculated as it was done previously in Eq. 39-40; however, the state-space formulation is slightly modified to allow for the calculation of the outputs to HydroDyn as:

$$
y_{2}=\left\{\begin{array}{c}
\bar{U}_{R} \\
U_{L} \\
\overline{\bar{U}}_{R} \\
\dot{U}_{L} \\
\bar{U}_{R} \\
\ddot{U}_{L}
\end{array}\right\}=\left\{\begin{array}{c}
\bar{U}_{R} \\
\hat{U}_{L}+U_{L 0}-U_{L 0 m} \\
\overline{\bar{U}}_{R} \\
\dot{U}_{L} \\
\ddot{\bar{U}}_{R} \\
\ddot{U}_{L}
\end{array}\right\}
$$

where the matrices now have the following meaning:

$$
\begin{aligned}
C_{2} & =\left[\begin{array}{ll}
0 & 0 \\
\Phi_{m} & 0 \\
0 & 0 \\
0 & \Phi_{m} \\
0 & 0 \\
-\Phi_{m} \Omega_{m}^{2} & -2 \Phi_{m} \zeta \Omega_{m}
\end{array}\right], D_{2}=\left[\begin{array}{ccccc}
T_{I} & 0 & 0 & 0 & 0 \\
\bar{\Phi}_{R} T_{I} & 0 & 0 & 0 & 0 \\
0 & T_{I} & 0 & 0 & 0 \\
0 & \bar{\Phi}_{R} T_{I} & 0 & 0 & 0 \\
0 & 0 & T_{I} & 0 & 0 \\
0 & 0 & \bar{\Phi}_{R} T_{I}-\Phi_{m} \tilde{M}_{m B} & \Phi_{m} \Phi_{m}^{T} & 0
\end{array}\right], \\
F_{Y 2} & =\left[\begin{array}{c}
0 \\
U_{L 0}-U_{L 0 m} \\
0 \\
0 \\
0 \\
\Phi_{m} \Phi_{m}^{T} F_{L g}
\end{array}\right]
\end{aligned}
$$

Finally, the element forces can be calculated as:

$$
\begin{aligned}
& \text { Element Inertia load: } F_{I}^{e}=M^{e} \ddot{U}^{e} \\
& \text { Element Static load: } F_{S}^{e}=K^{e} U^{e}=K^{e}\left[\hat{U}^{e}+U_{L 0}^{e}-U_{L 0 m}^{e}\right]
\end{aligned}
$$

with the element node DOFs expressed as:

$$
U^{e}=\hat{U}^{e}+U_{L 0}^{e}-U_{L 0 m}^{e}
$$

where the SIM decomposition is still used with $\hat{U}^{e}$, denoting the time-varying components of the elements nodes' displacements and $U_{L 0}^{e}$ and $U_{L 0 m}^{e}$ are derived from the parent $U_{L 0}$ and $U_{L 0 m}$ arrays of displacements, respectively. 


\section{Known Limitations and Future Work}

The following list contains known current limitations in the code:

- Tight coupling is not yet supported.

- Limited restraint capabilities are available (only clamped joints allowed).

- Only nontapered two-node Euler-Bernoulli (E-B) or Timoshenko (T) element formulations are available. (In the future, tapered E-B and tapered Timoshenko element formulations will be implemented.)

- Only circular members are permitted. (In the future, a generic cross section will be allowed.)

- The number of elements per member (NDiv) is constant throughout the structure.

- Internal matrices are not stored in sparse form, limiting the total number of possible nodes/DOFs to about 300/1800.

- Foundation (soil-structure interaction) modeling is not yet available.

- The dynamics system reduction is performed in the absence of external loading (e.g., hydrodynamic added mass).

- Gravitational loading does not impact the global substructure stiffness.

- Loads (gravitational, inertial, hydrodynamic) can only be applied as concentrated loads at element nodes; distributed loads (per unit length) are not yet supported.

- The overlap of multiple members connected to a single joint is not modeled with superelements.

- Member-level outputs are only available for up to nine nodes of up to nine members (although the OutAll flag can generate further outputs at the member end nodes).

- No graphics/animation capability is yet available to visualize the substructure geometry, modes, motion, and loads. 


\section{References}

Chapra, S. C.; Canale, R. P. (2010). Numerical Methods for Engineers, McGraw-Hill Science/Engineering/Mat, $6^{\text {th }}$ Edition, 960 pages.

Craig, R.; Bampton, M. (1968). "Coupling of Substructures for Dynamic Analyses.” AIAA Journal, Vol. 6, No.7, July 1968, pp. 1313-1319.

Damiani, R.; Song, H. (2013). "A Jacket Sizing Tool for Offshore Wind Turbines within the Systems Engineering Initiative.” Offshore Technology Conference, Houston, TX, April 30-May 4, 2013.

Damiani, R.; Song, H.; Robertson, A.; Jonkman, J. (2013). "Assessing the Importance of Nonlinear Structural Characteristics in the Development of a Jacket Model for the Wind Turbine CAE Tool FAST." 32nd International Conference on Ocean, Offshore and Arctic Engineering (OMAE2013), Nantes, France; June 9-14, 2013.

Hurty, W.C. (1964). "On the Dynamic Analysis of Structural Systems Using Component Modes.” AIAA Paper No. 64-487, First AIAA Annual Meeting, Washington, DC, June 29-July $2,1964$.

Jonkman, J.M. (2013). "The New Modularization Framework for the FAST Wind Turbine CAE Tool." 51st AIAA Aerospace Sciences Meeting and 31st ASME Wind Energy Symposium, Grapevine, TX.

Jonkman, J.M.; Robertson, A.N.; and Hayman, G.J. (forthcoming). HydroDyn User's Guide and Theory Manual.. Golden, CO: National Renewable Energy Laboratory.

Panzer, H.; Hubele, J.; Eid, R.; Lohmann, B. (2009). Generating a Parametric Finite Element Model of a 3D Cantilever Timoshenko Beam Using Matlab. Technical Reports on Automatic Control, Vol. TRAC-4, November 2009, Technische Universitat Munchen.

Song, H.; Damiani, R.; Robertson, A.; Jonkman, J. (2013). "New Structural-Dynamics Module for Offshore Multimember Substructures within the Wind Turbine Computer-Aided Engineering Tool FAST." Preprint. Presented at the 23rd International Ocean, Offshore and Polar Engineering Conference - ISOPE 2013, 30 June - 5 July 2013, Anchorage, Alaska. 12 pp.; NREL Report No. CP-5000-58093.

Sprague, M. A.; Jonkman, J. M.; Jonkman, B. J. (2014). "FAST Modular Wind Turbine CAE Tool: Nonmatching Spatial and Temporal Meshes." Proceedings of the 32nd ASME Wind Energy Symposium, 13-17 January 2014, National Harbor, Maryland. American Society of Mechanical Engineers (ASME) New York, NY. 24 pp.; NREL Report No. CP-2C00-61839. Steinboeck, A.; Kugi, A.; Mang, H. (2013). "Energy-consistent shear coefficients for beams with circular cross sections and radially in homogeneous materials." International Journal of Solids and Structures, 50, no. 11-12, pp. 1859-1868, doi: 10.1016/j.jisolstr.2013.01.030. 


\section{Appendix A. OC4 Jacket Input File}

- SubDyn v1.01.x MultiMember Support Structure Input File

OC4 'Jacket' SubStructure Input File. The grouted connection is simulated with an equivalent tubular beam of enhanced properties. RRD 10/15/2013

$\begin{array}{lll}\text { False } & \text { Echo } & \text { - Echo input data to "<rootname>.SD.ech" (flag) } \\ \text { "DEFAULT" } & \begin{array}{l}\text { SDdeltaT } \\ \text { IntMethod }\end{array} & \text { - Local Integration Step. If "default", the glue-code integration step will be used. } \\ \text { SttcSolve } & \text { - Solve dynamics about static equilibrium point }\end{array}$

(unavailable); 3= 2-node Timoshenko; 4= 2-node ...

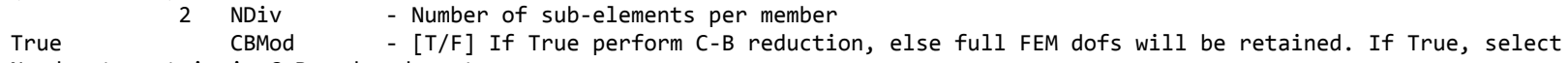
Nmodes to retain in $\mathrm{C}-\mathrm{B}$ reduced system.

$\begin{array}{lllll} & 8 & \text { Nmodes } & \text { - Number of internal modes to retain (ignored if CBMod=False). If Nmodes }=0 \text { - }->\text { Guyan } \\ \text { Reduction. } & 1 & \text { JDampings } & \text { - Damping Ratios for each retained mode (\% of critical) If Nmodes }>0 \text {, list Nmodes structural }\end{array}$ damping ratios for each retained mode ....

-.-- STRUCTURE JOINTS: joints connect structure members ( Hydrodyn Input File)-.-

$\begin{array}{llll} & \text { NJoints } & - \text { Number of joints }(-) & \text { JointYss } \\ \text { JointID } & \text { JointXss } & \text { JointZss } & \text { [Coordinates of Member joints in SS-Coordinate }\end{array}$

\section{System ]}

(-)

2

3

6

7

8

9

12

16

21

22

24

\section{$(m)$}

6.00000

6.00000

5.96700

5.93900

5.33300

$-6.00000$

$-6.00000$

$-5.96700$

$-5.93900$

$-5.33300$

$-6.00000$

$-6.00000$

$-5.96700$

$-5.93900$

$-5.33300$

6.00000

6.00000

5.96700

5.93900

5.33300

4.82000

4.38500

4.01600

4.00000

$-4.82000$

$-4.38500$

$-4.01600$

$-4.00000$

$-4.82000$

$-4.38500$

$-4.01600$

$-4.00000$

4.82000

4.38500

4.01600

4.00000

5.62000

$-5.62000$

0.00000

0.00000

5.06400

$-5.06400$

0.00000

0.00000

4.59200

$-4.59200$

0.00000

0.00000

4.19300

$-4.19300$

0.00000

0.00000

\section{(m)}

6.00000

6.00000

5.96700

5.93900

5.33300

6.00000

6.00000

5.96700

5.93900

5.33300

$-6.00000$

$-6.00000$

$-5.96700$

$-5.93900$

$-5.33300$

$-6.00000$

$-6.00000$

$-5.96700$

$-5.93900$

$-5.33300$

4.82000

4.38500

4.01600

4.00000

4.82000

4.38500

4.01600

4.00000

$-4.82000$

$-4.38500$

$-4.01600$

$-4.00000$

$-4.82000$

$-4.38500$

$-4.01600$

$-4.00000$

0.00000

0.00000

5.62000

$-5.62000$

0.00000

0.00000

5.06400

$-5.06400$

0.00000

0.00000

4.59200

$-4.59200$

0.00000

0.00000

4.19300

$-4.19300$ (m)

$-45.50000$

$-45.00000$

$-44.00100$

$-43.12700$

$-24.61400$

$-45.50000$

$-45.00000$

$-44.00100$

$-43.12700$

$-24.61400$

$-45.50000$

$-45.00000$

$-44.00100$

$-43.12700$

$-24.61400$

$-45.50000$

$-45.00000$

$-44.00100$

$-43.12700$

$-24.61400$

$-8.92200$

4.37800

15.65100

16.15000

$-8.92200$

4.37800

15.65100

16.15000

$-8.92200$

4.37800

15.65100

16.15000

$-8.92200$

4.37800

15.65100

16.15000

$-33.37300$

$-33.37300$

$-33.37300$

$-33.37300$

$-16.37100$

$-16.37100$

$-16.37100$

$-16.37100$

$-1.95800$

$-1.95800$

$-1.95800$

$-1.95800$

10.26200

10.26200

10.26200

10.26200 


$\begin{array}{rrrr}53 & 4.00000 & 4.00000 & 20.15000 \\ 54 & -4.00000 & 4.00000 & 20.15000 \\ 55 & 4.00000 & -4.00000 & 20.15000 \\ 56 & -4.00000 & -4.00000 & 20.15000 \\ 57 & 6.00000 & -6.00000 & -49.50000 \\ 58 & 6.00000 & 6.00000 & -49.50000 \\ 59 & -6.00000 & -6.00000 & -49.50000 \\ 60 & -6.00000 & 6.00000 & -49.50000 \\ 61 & 6.00000 & -6.00000 & -50.00100 \\ 62 & 6.00000 & 6.00000 & -50.00100 \\ 63 & -6.00000 & -6.00000 & -50.00100 \\ 64 & -6.00000 & 6.00000 & -50.00100\end{array}$

. BASE REACTION JOINTS: 1/0 for Locked/Free DOF @ each Reaction Node

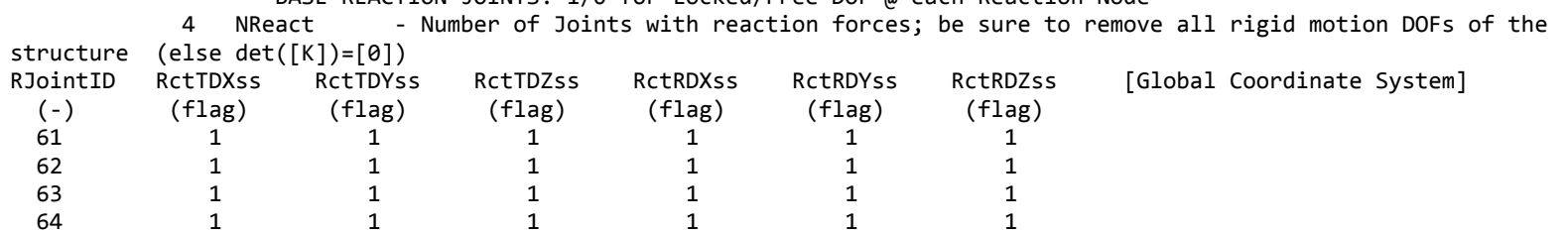

-..-- INTERFACE JOINTS: 1/0 for Locked (to the TP)/Free DOF @each Interface Joint (only Locked-to-TP implemented thus

\begin{tabular}{|c|c|c|c|c|c|c|}
\hline $\begin{array}{c}\text { IJointID } \\
(-)\end{array}$ & $\begin{array}{c}\text { ItfTDXss } \\
(\text { flag })\end{array}$ & $\begin{array}{c}\text { ItfTDYss } \\
\text { (flag) }\end{array}$ & $\begin{array}{c}\text { ItfTDZss } \\
(\text { flag })\end{array}$ & $\begin{array}{c}\text { ItfRDXss } \\
\text { (flag) }\end{array}$ & $\begin{array}{c}\text { ItfRDYss } \\
\text { (flag) }\end{array}$ & $\begin{array}{l}\text { ItfRDZss } \\
(\text { flag })\end{array}$ \\
\hline 24 & 1 & 1 & 1 & 1 & 1 & 1 \\
\hline 28 & 1 & 1 & 1 & 1 & 1 & 1 \\
\hline 32 & 1 & 1 & 1 & 1 & 1 & 1 \\
\hline 36 & 1 & 1 & 1 & 1 & 1 & 1 \\
\hline 53 & 1 & 1 & 1 & 1 & 1 & 1 \\
\hline 54 & 1 & 1 & 1 & 1 & 1 & 1 \\
\hline 55 & 1 & 1 & 1 & 1 & 1 & 1 \\
\hline 56 & 1 & 1 & 1 & 1 & 1 & 1 \\
\hline
\end{tabular}

8 NInterf - Number of interface joints locked to the Transition Piece (TP): be sure to remove all

$\begin{array}{llll} & 112 & \text { NMembers } & \text { Number of frame members } \\ \text { MemberID } & \text { MJointID1 MJointID2 MPropsetID1 MPropsetID2 COSMID }\end{array}$

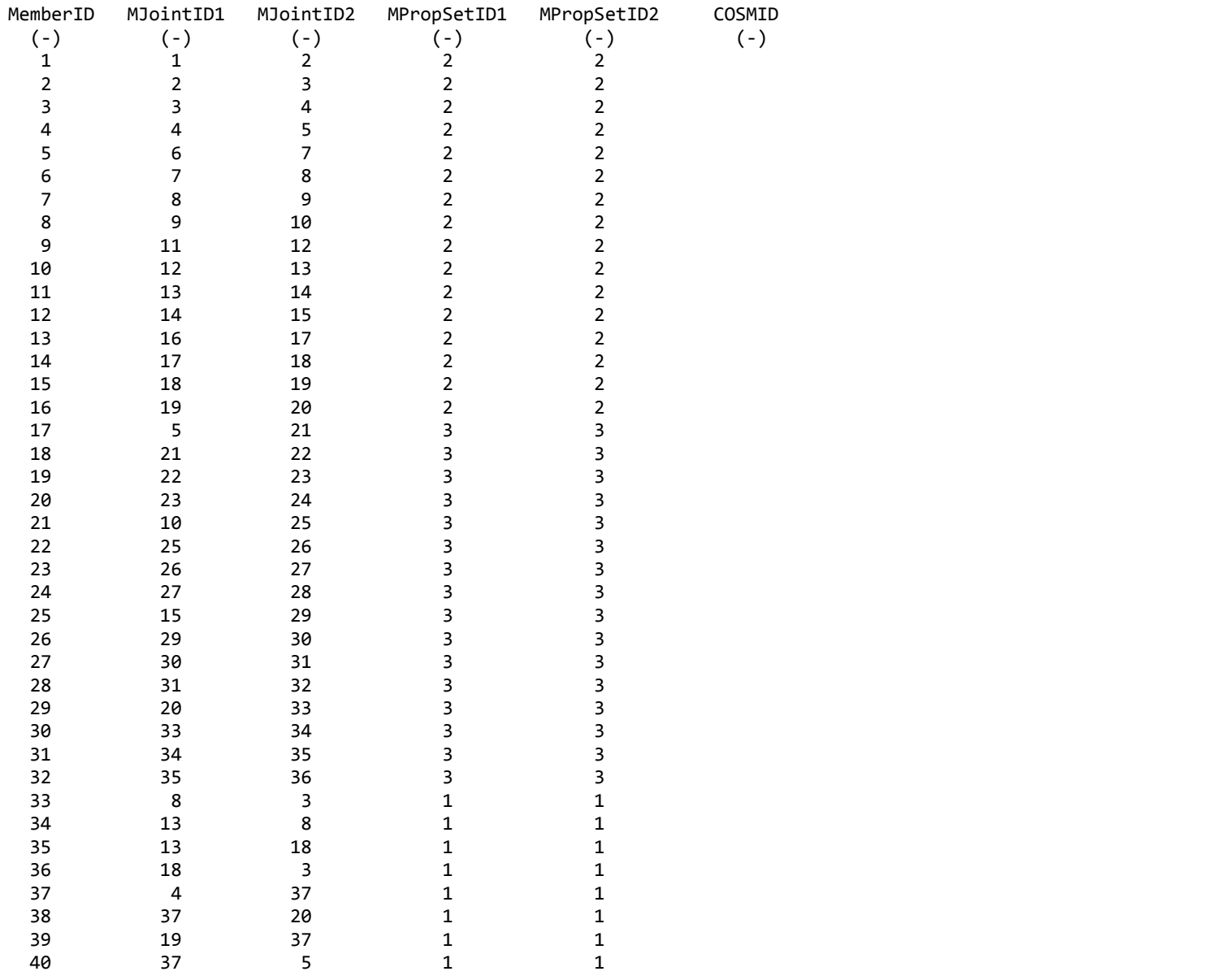




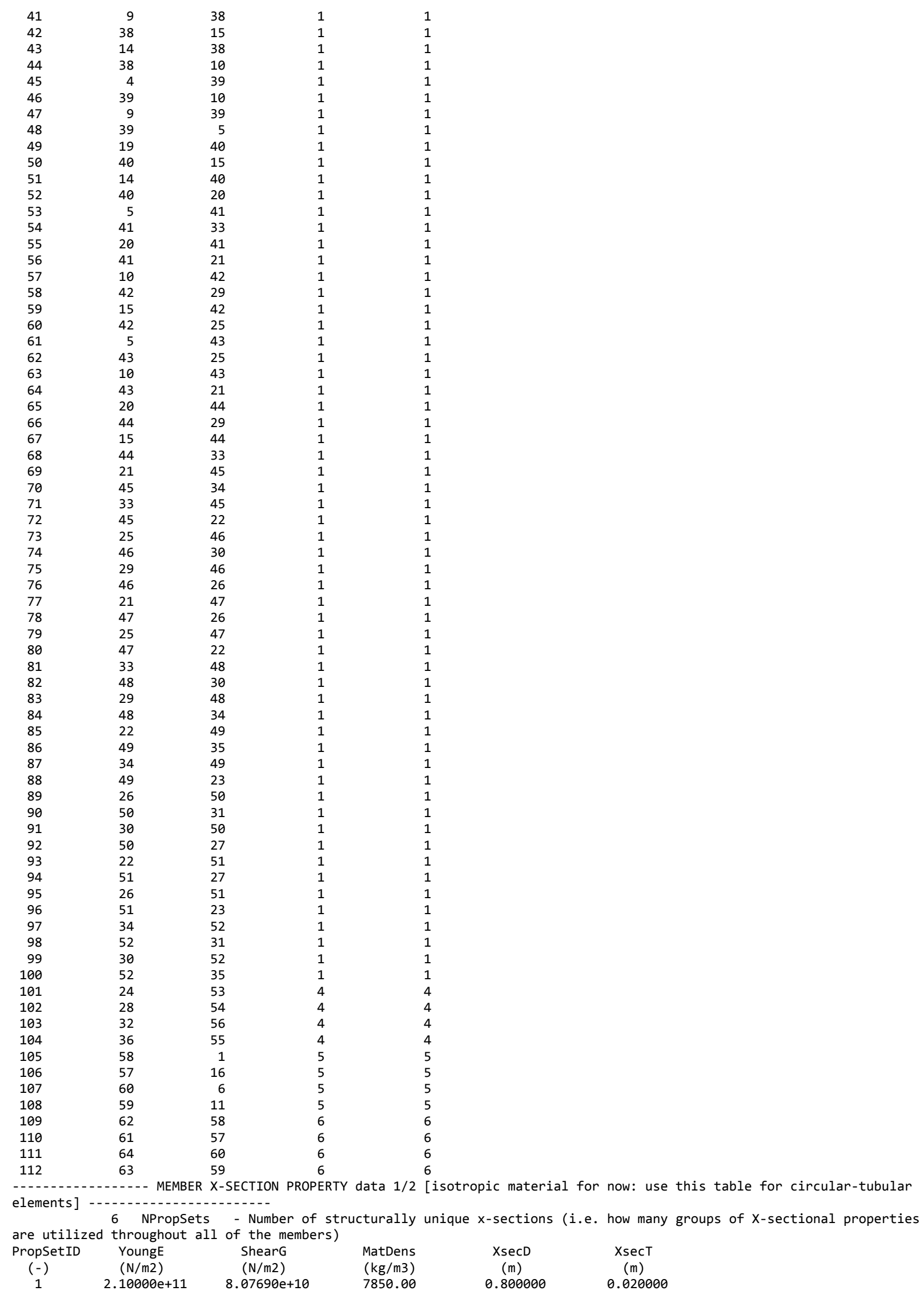




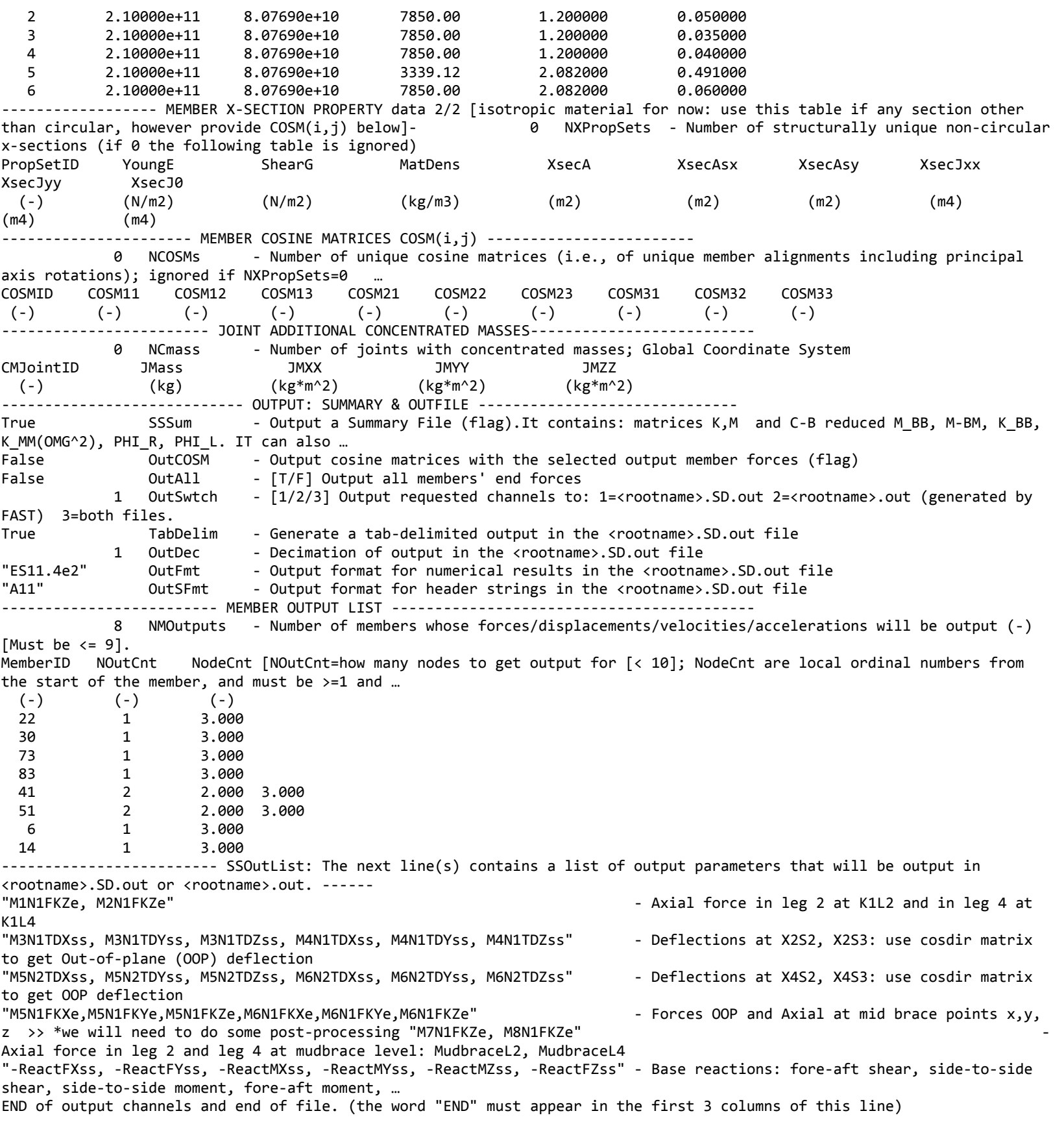




\section{Appendix B. OC4 Jacket Driver File}

SubDyn Driver file for Unit TestFrame.

Compatible with SubDyn v1.00.00

TRUE Echo - Echo the input file data (flag)

ENVIRONMENTAL CONDITIONS -

WtrDpth - Water Depth $(m)$ positive value

"Test04\Test04.txt" SubDyn SDInputFile

"Test04\Test04" OutRootName

600 NSteps - Number of time steps in the simulations (-)

0.005 TimeInterval - TimeInterval for the simulation (sec)

$0.0 \quad 0.0 \quad 18.15$ TP_RefPoint - Location of the TP reference point in global coordinates (m)

0.0 SubRotatez - Rotation angle of the structure geometry in degrees about the global $\mathrm{Z}$ axis.

(1) INPUTS -

1 InputsMod - Inputs model $\{0$ : all inputs are zero for every timestep, 1: steadystate inputs, 2: read inputs from a file (InputsFile)\} (switch)

" " InputsFile - Name of the inputs file if InputsMod = 2

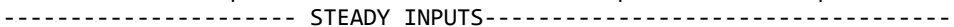

3.821E-02 1.656E-03 -4.325E-02 -1.339E-04 7.266E-02 -2.411E-03 uTPInSteady - input displacements and rotations (

$\mathrm{m}$, rads )

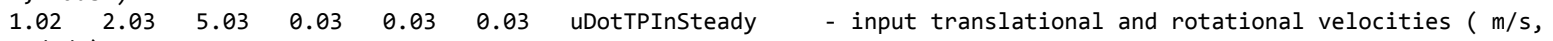

rads/s)

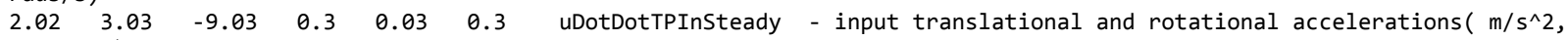

rads $/ \mathrm{s}^{\wedge} 2$ )

END of driver input file 


\section{Appendix C. List of Output Channels}

Table C-1 provides a list of all possible output parameters for the SubDyn module. The names are grouped by meaning, but can be ordered in the OUTPUT CHANNELS section of the SubDyn input file as the user sees fit. $M \alpha N \beta$, refers to node $\beta$ of member $\alpha$, where $\alpha$ is a number in the range [1,9] and corresponds to row $\alpha$ in the MEMBER OUTPUT LIST table (see Section 2.3.11) and $\beta$ is a number in the range [1,9] and corresponds to node $\beta$ in the NodeCnt list of that table entry.

Some outputs are in the SS reference coordinate system (global inertial-frame coordinate system), and end with the suffix 'ss'; others refer to the local (member) reference system and they have suffixes 'Xe', 'Ye', or 'Ze' (see Section 7).

Table C-1. Possible SubDyn Output Channels

\begin{tabular}{|c|c|c|}
\hline Channel Name(s) & Units & Description \\
\hline \multicolumn{3}{|c|}{ Base and Interface Reaction Loads } \\
\hline $\begin{array}{l}\text { ReactFXss, ReactFYss, ReactFZss, } \\
\text { ReactMXss, ReactMYss, ReactMZss, }\end{array}$ & $\begin{array}{l}(\mathrm{N}),(\mathrm{N}),(\mathrm{N}),(\mathrm{N} \cdot \mathrm{m}) \\
(\mathrm{N} \cdot \mathrm{m}),(\mathrm{N} \cdot \mathrm{m})\end{array}$ & $\begin{array}{l}\text { Total base reaction forces and } \\
\text { moments at the }(0 ., 0 .,- \text { WtrDpth) } \\
\text { location in SS coordinate system }\end{array}$ \\
\hline $\begin{array}{l}\text { IntfFXss, IntfFYss, IntfFZss, } \\
\text { IntfMXss, IntfMYss, IntfMZss, }\end{array}$ & $\begin{array}{l}(\mathrm{N}),(\mathrm{N}),(\mathrm{N}),(\mathrm{N} \cdot \mathrm{m}) \\
(\mathrm{N} \cdot \mathrm{m}),(\mathrm{N} \cdot \mathrm{m})\end{array}$ & $\begin{array}{l}\text { Total interface reaction forces and } \\
\text { moments at the TP reference } \\
\text { point (platform reference point) } \\
\text { location in SS coordinate system }\end{array}$ \\
\hline \multicolumn{3}{|c|}{ Interface Kinematics } \\
\hline $\begin{array}{l}\text { IntfTDXss, IntfTDYss, IntfTDZss, } \\
\text { IntfRDXss, IntfRDYss IntfRDZss }\end{array}$ & $\begin{array}{l}(\mathrm{m}),(\mathrm{m}),(\mathrm{m}),(\mathrm{rad}) \\
(\mathrm{rad}),(\mathrm{rad})\end{array}$ & $\begin{array}{l}\text { Displacements and rotations of } \\
\text { the TP reference point (platform } \\
\text { reference point) location in SS } \\
\text { coordinate system }\end{array}$ \\
\hline $\begin{array}{l}\text { IntfTAXss, IntfTAYss, IntfTAZss, } \\
\text { IntfRAXss, IntfRAYss IntfRAZss }\end{array}$ & $\begin{array}{l}\left(\mathrm{m} / \mathrm{s}^{\wedge} 2\right),\left(\mathrm{m} / \mathrm{s}^{\wedge} 2\right) \\
\left(\mathrm{m} / \mathrm{s}^{\wedge} 2\right),\left(\mathrm{rad} / \mathrm{s}^{\wedge} 2\right) \\
\left(\mathrm{rad} / \mathrm{s}^{\wedge} 2\right),\left(\mathrm{rad} / \mathrm{s}^{\wedge} 2\right)\end{array}$ & $\begin{array}{l}\text { Translational and rotational } \\
\text { accelerations of the TP reference } \\
\text { point (platform reference point) } \\
\text { location in SS coordinate system }\end{array}$ \\
\hline \multicolumn{3}{|c|}{ Modal Parameters } \\
\hline SSqm01-SSqm99 & $(-)$ & $\begin{array}{l}\text { C-B modal variables (up to first } \\
\text { 99) }\end{array}$ \\
\hline SSqmd01-SSqmd99 & $(1 / s)$ & $\begin{array}{l}\text { First time-derivatives of C-B } \\
\text { modal variables (up to first } 99 \text { ) }\end{array}$ \\
\hline SSqmdd01-SSqmdd99 & $\left(1 / s^{\wedge} 2\right)$ & $\begin{array}{l}\text { Second time-derivatives of C-B } \\
\text { modal variables (up to first } 99 \text { ) }\end{array}$ \\
\hline \multicolumn{3}{|c|}{ Node Kinematics } \\
\hline 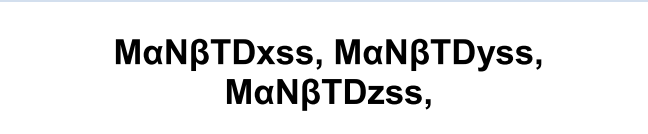 & (m) & $\begin{array}{c}\text { Nodal displacement of MaN } \beta \text { (up } \\
\text { to } 81 \text { designated locations) in SS } \\
\text { coordinate system }\end{array}$ \\
\hline
\end{tabular}




\begin{tabular}{|c|c|c|}
\hline 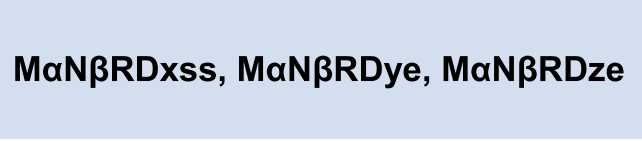 & (rad) & $\begin{array}{l}\text { Nodal rotations of MaN } \beta \text { (up to } 81 \\
\text { designated locations) in member } \\
\text { local coordinate system }\end{array}$ \\
\hline 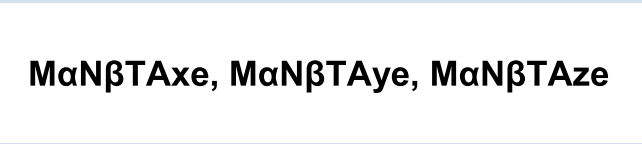 & $\left(\mathrm{m} / \mathrm{s}^{2}\right)$ & $\begin{array}{l}\text { Nodal accelerations of MaN } \beta \text { (up } \\
\text { to } 81 \text { designated locations) in } \\
\text { member local coordinate system }\end{array}$ \\
\hline 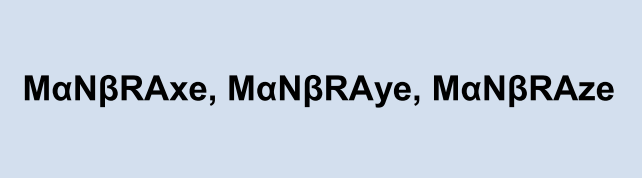 & $\left(\mathrm{rad} / \mathrm{s}^{2}\right)$ & $\begin{array}{l}\text { Nodal rotational accelerations of } \\
\text { MaN } \beta \text { (up to } 81 \text { designated } \\
\text { locations) in member local } \\
\text { coordinate system }\end{array}$ \\
\hline \multicolumn{3}{|c|}{ Node Forces and Moments } \\
\hline 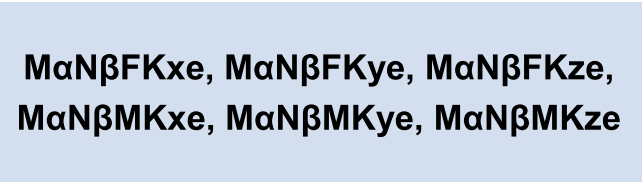 & $\begin{array}{l}(\mathrm{N}),(\mathrm{N}),(\mathrm{N}),(\mathrm{N} \cdot \mathrm{m}) \\
(\mathrm{N} \cdot \mathrm{m}),(\mathrm{N} \cdot \mathrm{m})\end{array}$ & $\begin{array}{l}\text { Static (elastic) component of } \\
\text { reaction forces and moments at } \\
\text { MaN } \beta \text { along local member } \\
\text { coordinate system }\end{array}$ \\
\hline 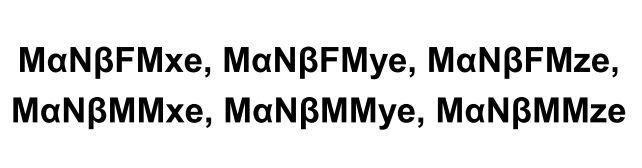 & $\begin{array}{c}(\mathrm{N}),(\mathrm{N}),(\mathrm{N}),(\mathrm{N} \cdot \mathrm{m}) \\
(\mathrm{N} \cdot \mathrm{m}),(\mathrm{N} \cdot \mathrm{m})\end{array}$ & $\begin{array}{l}\text { Dynamic (inertial) component of } \\
\text { reaction forces and moments at } \\
\text { MaN } \beta \text { along local member } \\
\text { coordinate system }\end{array}$ \\
\hline
\end{tabular}




\section{Appendix D. Compiling Stand-Alone SubDyn}

See the FAST documentation for instructions on how to compile SubDyn coupled to FAST.

Future versions of the manual will include compiling instructions for building the stand-alone SubDyn program. 


\section{Appendix E. Major Changes in SubDyn}

When first released, SubDyn (v0.4) was included as an undocumented feature of FAST v8 and packaged as a stand-alone archive. Since v0.4, SubDyn has been well integrated into FAST v8 and the stand-alone form is also available. This appendix outlines significant modifications to SubDyn made since v0.4. Following are the main changes that the user may notice, but for more information, refer to the changelog.txt text file within the official archive.

\section{V1.01.01a-rrd (September 2014)}

Version 1.01.01a-rrd integrates with the FAST v8 software v8.09.00a-bjj.

- Finite-element eigenvalue bug fixes: the full system eigenvalues were incorrectly reported in the summary file, although with no further consequences on the results. This bug is now fixed.

- Shear area correction factor improvement: the shear area correction factor in the Timoshenko treatment is now aligned with Steinboeck et al. (2013).

- The formulation for the TP reaction has been rearranged to adhere to the theory manual, with no consequences on the output results.

\section{V1.01.00a-rrd (June 2014)}

Version 1.00.01a-rrd integrates with the FAST v8 software v8.08.00c-bjj.

The new implementation has well-defined data exchange interfaces (following the FAST modularization framework) that should make integration of SubDyn into other multiphysics software packages much simpler.

Several improvements and bug fixes have been implemented since version v0.4 and the module has undergone an extensive verification effort with good results.

- Eigensolver bug fixes: the LAPACK solver proved to be unstable in single precision, and now it is solely run in double precision, regardless of the precision used in other parts of the code.

- The input file format has changed. Please refer to the sample input file in Appendix A and the following notes:

- First header line has been removed.

- Simulation Control Section:

- SDeltaT: The 'DEFAULT' keyword (in place of 0.0) is now used to indicate that the glue-code time step will be used for time integration by SubDyn.

- IntMethod: Allowed values are now 1-4 (in place of 0-3).

- SttcSolve: New flag introduced. If set to TRUE, the static-improvement method (SIM) will be used. 
- FEA and Craig-Bampton Parameters Section:

- In v0.4, the damping coefficients had to be specified for all retained CraigBampton modes, or just once for all the modes (if $\boldsymbol{C B M o d}=$ FALSE). In this version, the user can input any number of damping coefficients. In case the number of retained C-B modes (NModes) is larger than the input number of damping coefficients (JDampings), the last damping value will be replicated for all the remaining modes.

- Base Reaction Joints, Interface Joints, Member, and Member Cosine Matrices Sections:

- One line with units, below the headers, is expected in all the tables of the input file.

○ Output: Summary and Outfile Section:

- This section now also contains the parameters previously assigned under the Section titled "Output: Fast/Subdyn Output-File Variables"

- Some of the quantities in the summary file have been fixed. Some of the output matrices were, in fact, being output with wrong values because of an index mismatch. The new summary file is shorter and does not contain some of the $\mathrm{CB}$ method matrices, unless the compiler directive, DEBUG, is set.

- SIM. This new implementation helps minimize the number of needed modes to capture the contribution of certain loads (such as static gravity and buoyancy loads or highfrequency loads transferred from the turbine). In the previous version, a large number of internal modes were needed to engage substructural modes excited by static and highfrequency forces. These modes are no longer needed and fewer modes can be retained while still achieving accurate results (see also Section 6). With SIM enabled, all modes that are not considered by the Craig-Bampton reduction are treated quasi-statically.

- There is now the possibility of retaining no internal C-B modes, thus relying solely only on SIM, in those cases where the substructure's first eigenfrequencies are much higher than the expected energy-containing modes of the entire system.

- The coupling of SubDyn within FAST now includes full hydro-elastic coupling with the HydroDyn hydrodynamics module. 